\title{
Higher-order acoustic diffraction by edges of finite thickness
}

\author{
Dezhang $\mathrm{Chu}^{\mathrm{a})}$ and Timothy K. Stanton \\ Department of Ocean Physics and Engineering, Woods Hole Oceanographic Institution, Woods Hole, \\ Massachusetts 02543-1053 \\ Allan D. Pierce \\ Aerospace and Mechanical Engineering, Boston University, Boston, Massachusetts 02215
}

(Received 6 September 2005; revised 9 April 2007; accepted 9 August 2007)

\begin{abstract}
A cw solution of acoustic diffraction by a three-sided semi-infinite barrier or a double edge, where the width of the midplanar segment is finite and cannot be ignored, involving all orders of diffraction is presented. The solution is an extension of the asymptotic formulas for the double-edge second-order diffraction via amplitude and phase matching given by Pierce [A. D. Pierce, J. Acoust. Soc. Am. 55, 943-955 (1974)]. The model accounts for all orders of diffraction and is valid for all $k w$, where $k$ is the acoustic wave number and $w$ is the width of the midplanar segment and reduces to the solution of diffraction by a single knife edge as $w \rightarrow 0$. The theory is incorporated into the deformed edge solution [Stanton et al., J. Acoust. Soc. Am. 122, 3167 (2007)] to model the diffraction by a disk of finite thickness, and is compared with laboratory experiments of backscattering by elastic disks of various thicknesses and by a hard strip. It is shown that the model describes the edge diffraction reasonably well in predicting the diffraction as a function of scattering angle, edge thickness, and frequency. (C) 2007 Acoustical Society of America.
\end{abstract}

[DOI: $10.1121 / 1.2783001]$

PACS number(s): 43.20.El, 43.30.Ft [AJMD]

Pages: $3177-3194$

\section{INTRODUCTION}

To study the scattering by a seafloor covered by shells, laboratory measurements of free-field backscattering by sea shells and a variety of machined circular disks with different sizes, thicknesses, and material properties were made (Stanton and Chu, 2004). Qualitative similarities were observed between the scattering by the edges of the disks and the edges of the shells (especially the sand dollar) (Stanton and Chu, 2004). A major challenge remains for quantitatively describing acoustic diffraction by edges that occur in nature, which are deformed, of finite length, and are composed of a complex material such as one with elastic properties.

One special case of deformed edges of finite length concerning the problem of diffraction by circular disks has been studied intensively by many investigators. Sleator (1969) presented an exact solution based on the modal series solution of an oblate spheroid by letting the aspect ratio approach zero. The $\mathrm{T}$ matrix method, a formally exact analytical/ numerical approach, has been used to study the acoustic and electromagnetic scattering by circular disks (Kristensson and Waterman, 1982). A number of approximate approaches have also been developed. Keiffer et al. (1994), by using the Huygens wavelets approach, presented a discrete wedge assemblage (WA) model that was based on the exact and closed form solution of scattering by an infinitely long straight wedge in the time domain (Biot and Tolstoy, 1957). This method was first proposed by Medwin et al. (1982) by using the truncated time series from the solution to an infinitely long straight wedge to describe the diffraction by straight

\footnotetext{
${ }^{a)}$ Current address: NOAA-NMFS, Northwest Fisheries Science Center, Seattle, WA 98112. Electronic mail: dchu@whoi.edu
}

finite-length wedges. Svensson et al. (1999) extended the WA model to a more generalized integral form that can include the second-order diffraction by using the secondary edge sources. It was shown that a second-order diffracted component that circumnavigates the disk could be accurately included in the calculations and was calculated numerically. Recently, Li et al. (1998) presented a hybrid-iterative method to compute the scattering by a conducting circular disk. The model combined analytical and numerical approaches and employed a number of techniques including the vector wave eigenfunction expansion, the least-squares method, and the mode matching. A brief review of other approximate methods was also given in that paper including the physical optics (Rahmat-Samii, 1988), the physical theory of diffraction (Ufimtsev, 1962), the geometric theory of diffraction (Keller, 1962), and the method of moments (Duan et al., 1991).

All of the above-mentioned methods were applied to the problem of calculating the diffraction by circular disks with single knife edges [left drawing in Fig. 1(a)], i.e., disks of zero thickness. However, realistic cases may involve edges of finite thickness-that is, a double edge or truncated wedge geometry [middle drawing in Fig. 1(a) and the threedimensional geometry shown in Fig. 1(b)]. For example, in a recent paper, the scattering by elastic circular disks was studied for the purpose of understanding the underlying scattering mechanisms of benthic shells (Stanton and Chu, 2004). Although the disks were quite thin relative to their diameter, the value of $k w$, where $k$ is the acoustic wave number and $w$ is the thickness, was comparable to unity. In this scattering region, the thickness cannot be ignored and higher-order diffraction between the two closely spaced parallel edges along the perimeter of the disk must be taken into account [right drawing in Fig. 1(a)]. 

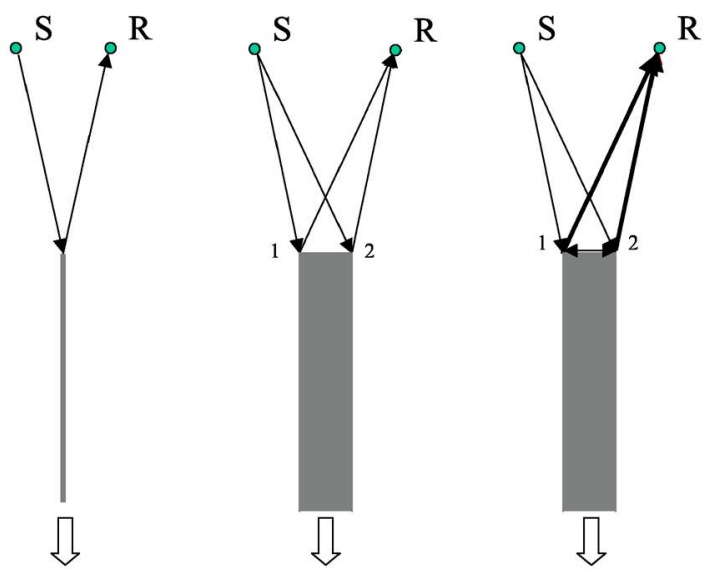

(a)

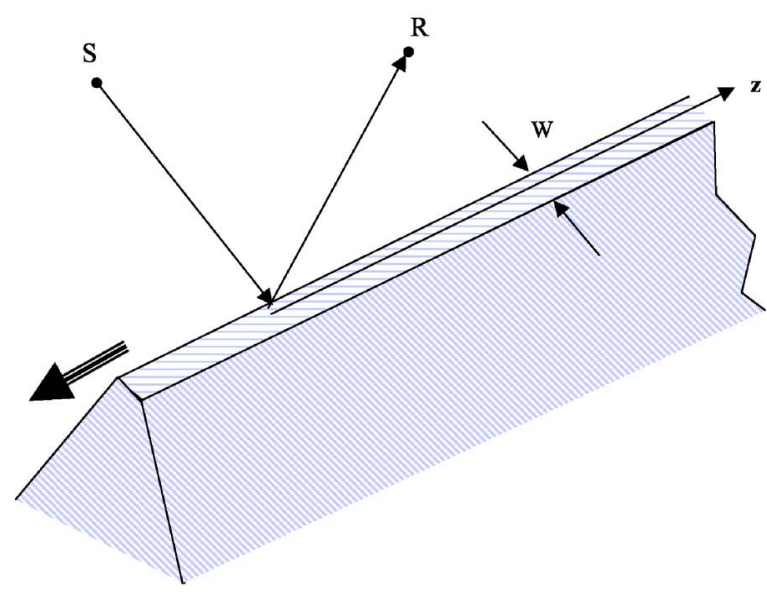

(b)

FIG. 1. (Color online) (a) 2D cross-sectional view of diffraction by infinitely long straight truncated wedges of two thicknesses. "S" and " $\mathrm{R}$ " indicate the locations of the point source and receiver, respectively. (b) Threedimensional (3D) view of diffraction by an infinitely long straight truncated wedge.

To demonstrate the necessity of including higher-order diffraction, we compare the experimental data [partial wave target strength (PWTS) of the leading edge, the portion of the disk closer to the transducers, i.e., " $A$ " on the left graph in Fig. 2] with the theoretical predictions (Fig. 2). The two diffraction models are the knife-edge single-diffraction model (solid) corresponding to the case illustrated in Fig. 1(a) (left) and the double-edge, first-order diffraction model (dashed) corresponding to the case also illustrated in Fig. 1(a) (middle). Both models are based on the asymptotic solution for an infinitely long straight wedge or a knife edge (Pierce, 1974) and are incorporated into the deformed wedge formulation (Stanton et al., 2007). Clearly, the singlediffraction model of a knife edge severely underestimates the edge diffraction from scattering angles between $30^{\circ}$ and $90^{\circ}$. It is well known that for an infinitely long screen of half space, or a knife edge, the diffraction approaches zero as the scattering angle (backscatter) approaches the edge-on incidence $\left(\theta=90^{\circ}\right.$ in Fig. 2). Another natural edge-diffraction model includes a simple summation of the first-order diffrac- tion by two wedges of right angle as shown as a dashed line in Fig. 2. This double-edge single-diffraction model provides a much better fit to the data, especially for the overall pattern, but it overestimates the overall level of diffraction by a few decibels. To better describe the diffraction by an edge of finite thickness, a more sophisticated model is desirable.

Predicting diffraction by disks of finite thickness faces two major challenges - one is to account for the deformation (circular curve) of the double edge and the other is to account for the multiple diffraction between the two closely spaced edges. The deformation can be accounted for with an approximate line integral approach and has been studied in a separate paper (Stanton et al., 2007). In this paper, higherorder diffraction between two infinitely long edges is studied and formulations are developed. The approach is based on a formulation published by Pierce (1974) where the secondorder diffraction is included for cases when $k w \gg 1$. In this paper, Pierce's approach is extended to include all orders of diffraction, in which a heuristic formula is used to analytically connect the strength of the virtual sources associated with the thickness between the thin (knife edge) and thick (finite thickness) disk solutions so that the solution can be used to estimate diffraction for all thicknesses. The solution, once incorporated into the deformed edge line integral and using empirically determined coefficients, is compared with the laboratory data involving machined elastic disks of various thicknesses as a function of orientation and disk thickness. Furthermore, the model predictions are also compared with the previously published experimental data involving the diffraction by a straight strip of finite width (Medwin et al., 1982). In deriving the high-order diffraction model, since we include only the first arrival (edge diffraction) from the pulse-compressed signal for each ping and the reflection coefficient for an infinite aluminum plane is close to unity, the influence of elasticity is ignored and a proposed higher-order diffraction model is based on the rigid wedge solution.

The paper is organized as follows: Section II defines the problem. Section III briefly reviews the diffraction by an infinitely long straight single wedge and examines the diffracted field across the reflection and shadow boundaries for the two extreme situations $r \rightarrow 0$ and $r \rightarrow \infty$. In Sec. IV, the higher-order diffraction model will be developed. Modeldata comparison and the subsequent discussions will be included in Sec. V. Finally, summaries and conclusions are provided in Sec. VI.

\section{DEFINITION OF THE DIFFRACTION PROBLEM}

The total field associated with the presence of a diffracting object is the sum of the incident $\left(p_{\text {inc }}\right)$, reflected $\left(p_{\text {ref }}\right)$, and total diffracted $\left(p_{\text {dif,tot }}\right)$ fields and can be expressed as

$$
p_{\text {tot }}=p_{\text {inc }}+p_{\text {ref }}+p_{\text {dif,tot }} \text {. }
$$

The incident and reflected fields can be determined easily based on the scattering geometry using the method of images (Biot and Tolstoy, 1957). For a single edge or wedge, the total diffracted field is solely due to the first-order (single) diffraction by the edge [Fig. 1(a), left]. However, for the diffraction by two parallel infinitely long edges (double 


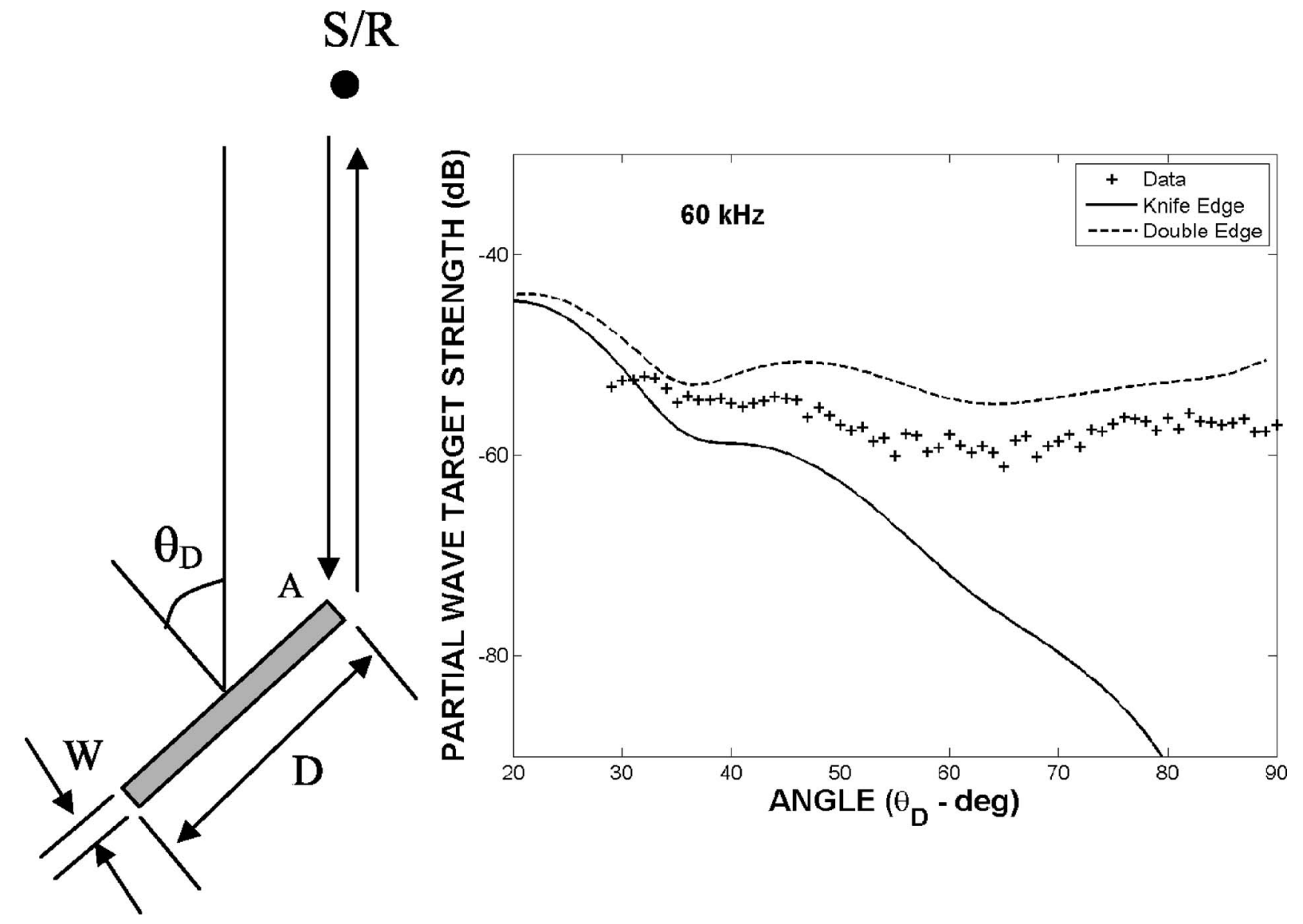

FIG. 2. Comparison between laboratory data and knife-edge diffraction model. The "+" symbol indicates measured diffraction by the leading-edge $(A)$ of an aluminum disk with a diameter of $8 \mathrm{~cm}(D)$ and thickness of $1.9 \mathrm{~mm}(W)$. The solid curve is the model prediction based on an infinitely long knife edge from Pierce (1974) and with the curvature of the edge accounted for using the method described in Stanton et al. (2007). The dashed curve is the sum of the first-order diffracted waves from the two right-angle wedges at $A$.

edge) or more generally, a truncated wedge [Fig. 1(b)], the total diffracted field is composed of the first-order diffraction from the two edges [Fig. 1(a), middle] and the higher-order diffraction between the edges [Fig. 1(a), right]

$$
p_{\text {dif,tot }}=\sum_{n=1}^{\infty} p_{\text {dif }, n},
$$

where $p_{\text {dif }, n}$ represents the $n$th order diffraction from the double edge. The second-order diffraction $p_{\text {dif, } 2}$ that corresponds to the ray path $S \rightarrow 1 \rightarrow 2 \rightarrow R$ or $S \rightarrow 2 \rightarrow 1 \rightarrow R$ [Fig. $1(\mathrm{a})$, right] is also referred to as "double diffraction" by a number of authors (Karp and Keller, 1960; Keller, 1962; Pierce, 1974; Medwin et al., 1982; Svensson et al., 1999). The reason why we use the terminology "second-order diffraction" instead of double diffraction is that double diffraction, when taken literally, could also imply first-order diffraction by edges 1 and 2 of the double edge [Fig. 1(a), middle] rather than second-order diffraction. In the following sections, we will derive an analytical expression for $p_{\text {dif,tot }}$ that includes all orders of diffraction from a double edge, or a truncated wedge.

\section{FIRST-ORDER DIFFRACTION}

\section{A. Background and Pierce's solution for first-order diffraction}

Diffraction of electromagnetic and acoustic waves by an infinitely long straight wedge (not truncated) has long been studied and can be traced back to Sommerfeld (1954), who, in 1896, first presented the exact solution in the frequency domain due to a plane incident wave for a knife edge (wedge angle of $\theta_{w}=2 \pi$, where $\theta_{w}$ is defined in Fig. 3) and suggested that the solution for a wedge with an arbitrary wedge angle can be derived based on the same principle. The diffraction by wedges for a cylindrical incident wave was presented by Carslaw $(1899,1920)$. Later on, MacDonald provided an exact solution, also in the frequency domain, for a rigid wedge due to a cylindrical source (MacDonald 1902) and a point source (MacDonald 1915) in the form of a contour integral. Biot and Tolstoy (1957), using the method of normal coordinates, presented an exact impulse solution in closed form for a rigid wedge due to a point source involving only elementary functions. Applications and developments based on Biot and Tolstoy's (BT) solution have been reported by many investigators (Medwin et al., 1982; Kinney et al., 1983; Daneshvar and Clay, 1987; Chu, 1989; Clay et al., 1993; Li et al., 1994; Keiffer et al., 1994; Medwin and Clay, 


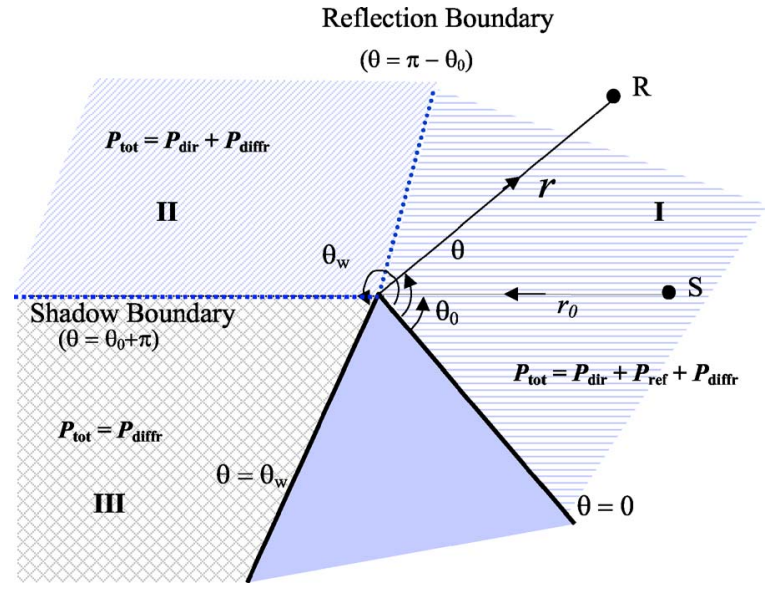

FIG. 3. (Color online) Geometry illustrating different diffraction regions associated with diffraction by an infinitely long straight wedge. 2D crosssectional view.

1998; Feuillade et al., 2004). At almost the same time when Biot and Tolstoy published their point source solution, in a much less referenced work by Friedlander (1958), an exact two-dimensional (2D) solution due to a line source in the time domain involving only the elementary functions was also presented. A variety of solutions using other methods and focusing on other types of applications have also been published (Keller et al., 1956; Keller, 1957, 1962; Ufimtsev, 1962; Morse and Ingard, 1968; Pierce, 1974, 1981; Tolstoy and Clay, 1987; Tolstoy, 1989a,b; Davis and Scharstein, 1997; Menounou et al., 2000).

We choose to begin with a solution that originated in Pierce (1974) and was given in a complete form in Pierce (1981). Because of the limited bandwidth of the acoustic system used in our experiment, this frequency domain $\mathrm{cw}$ solution is appropriate for our application. In addition, the spectral representation is a convenient analytical form that can be manipulated easily and extended to include all orders of diffraction. The integral form of a spectral representation of the exact solution of the diffracted wave from an infinitely rigid wedge (Fig. 3) due to a point source is (Pierce, 1981, pp. 479-489)

$$
p_{\mathrm{dif}}=-\frac{S \sin \nu \pi}{2 \theta_{w}} \sum_{ \pm} \int_{-\infty}^{\infty} \frac{e^{i k R(\pi-i s)}}{R(\pi-i s)} F_{s}\left(s, \theta \pm \theta_{0}\right) d s,
$$

where $S$ is the source strength, $\nu=\pi / \theta_{w}$ is the wedge parameter, and

$$
F_{s}(s, \vartheta)=\frac{\cos \nu \pi-\cos \nu \vartheta \cos \nu s}{(\cosh \nu s-1)^{2}+2(\cosh \nu s-1)(1-\cos \nu \vartheta \cos \nu \pi)+(\cos \nu \pi-\cos \nu \vartheta)^{2}},
$$

$$
\begin{aligned}
R(\pi-i s) & =\left(r^{2}+r_{0}^{2}-2 r r_{0} \cos (\pi-i s)+\left(z-z_{0}\right)^{2}\right)^{1 / 2} \\
& =\left(r^{2}+r_{0}^{2}+2 r r_{0} \cosh s+\left(z-z_{0}\right)^{2}\right)^{1 / 2} .
\end{aligned}
$$

Without loss of generality, we assume $\nu=\pi / \theta_{w}<1$ and 0 $\leqslant \theta_{0} \leqslant \pi$ in our analysis throughout the paper.

Although Eqs. (2a)-(2c) were originally derived using a contour integral, they can also be derived from the exact impulse solution given by Biot and Tolstoy (1957), which is based on the method of normal coordinates (see the Appendix). Equations (2a)-(2c) and (A10) are exactly the same. The exact $\mathrm{cw}$ solution for wave propagation in a $2 \mathrm{D}$ waveguide based on the normal coordinates was provided by Tolstoy and Clay (1987). The integral variable $s$ represents the imaginary part of a complex angle $\xi=\pi-i s$ as described in Pierce (1981, p. 489). When $\xi$ is a real number, it represents the angle between the receiver and the source images (Pierce, 1981; Biot and Tolstoy, 1957). For $k R(s)$ much greater than unity, Eq. (2a) reduces to a simpler but approximate form. Following the procedures outlined in Pierce (1981), the approximate solution can be expressed as

$$
p_{\text {dif }}=S \frac{e^{i k L}}{L}\left\{D_{+}+D_{-}\right\}=S \frac{e^{i k L}}{L} D_{\Sigma}, \quad k R \gg 1,
$$

where $D_{\Sigma} \equiv D_{+}+D_{-}$, will be referred to as "diffraction amplitude" throughout the paper and the distance

$$
L=\left[\left(r+r_{0}\right)^{2}+\left(z-z_{0}\right)^{2}\right]^{1 / 2}
$$

represents the shortest distance connecting the point source, the apex of the wedge, and the point receiver. The diffraction function $D_{ \pm}$is

$$
D_{ \pm}=\frac{e^{i \pi / 4}}{\sqrt{2}} \frac{\sin \nu \pi}{\left[1-\cos \nu \pi \cos \nu\left(\theta \pm \theta_{0}\right)\right]^{1 / 2}} A_{D}\left[\Gamma M_{\nu}\left(\theta \pm \theta_{0}\right)\right]
$$

where

$$
\begin{aligned}
& \Gamma=\sqrt{\frac{2 r r_{0}}{\lambda L}}, \\
& M_{\nu}(\vartheta)=\frac{\cos \nu \pi-\cos \nu \vartheta}{\nu(1-\cos \nu \pi \cos \nu \vartheta)^{1 / 2}},
\end{aligned}
$$

and $A_{D}(X)$ is the diffraction integral (Pierce, 1974, 1981),

$$
\begin{aligned}
A_{D}(X) & =\frac{1}{\pi \sqrt{2}} \int_{-\infty}^{\infty} \frac{e^{-u^{2}} d u}{\sqrt{\pi / 2} X-e^{-i \pi / 4} u} \\
& =\operatorname{sign}(X)[f(|X|)-i g(|X|)],
\end{aligned}
$$

where the functions $f(X)$ and $g(X)$ are auxiliary Fresnel functions defined in Abramowitz and Stegun (1971, p. 111). For small $X$, we have 


$$
\begin{aligned}
& f(X)=\frac{1}{2}-\frac{\pi}{4} X^{2}+\cdots, \\
& g(X)=\frac{1}{2}-X+\frac{\pi}{4} X^{2}-\cdots
\end{aligned}
$$

For large $X$, we have

$$
\begin{aligned}
& f(X)=\frac{1}{\pi X}-\frac{3}{\pi^{3} X^{5}}+\cdots, \\
& g(X)=\frac{1}{\pi^{2} X^{3}}-\frac{15}{\pi^{4} X^{7}}+\cdots .
\end{aligned}
$$

For $|X|>2$, the errors introduced by only keeping the leading term in Eqs. (8a) and (8b) are less than $1 \%$.

If $\Gamma \gg 1$ and the receiver is not too close to either the shadow boundary $\left(\theta=\pi+\theta_{0}\right.$ for $\theta_{0} \leqslant \theta_{w}-\pi$ or $\theta=\theta_{0}-\pi$ for $\left.\theta_{0} \geqslant \pi\right)$ or the reflection boundary $\left(\theta=\pi-\theta_{0}\right.$ for $\theta_{0} \leqslant \pi$ or $\theta=2 \theta_{w}-\pi+\theta_{0}$ for $\theta_{0} \geqslant \pi$ ) (Fig. 3), an asymptotic solution can be obtained by keeping only the first term of $f(X)$ and ignoring $g(X)$,

$$
\begin{aligned}
D_{\Sigma}= & \left(\frac{1}{\pi \Gamma}\right) \frac{e^{i \pi / 4}}{\sqrt{2}} \nu \sin \nu \pi\left[\frac{1}{\cos \nu \pi-\cos \nu\left(\theta+\theta_{0}\right)}\right. \\
& \left.+\frac{1}{\cos \nu \pi-\cos \nu\left(\theta-\theta_{0}\right)}\right] .
\end{aligned}
$$

The above-presented representation can also be obtained by applying the method of stationary phase directly to Eq. (2a)-(2c). The diffraction factor given by Eq. (9) is the same as that given by Keller (1962) using the geometric theory of diffraction (GTD), and by Morse and Ingard (1968) by directly solving the wave equation.

\section{B. Special limiting cases of first-order diffraction}

In Pierce's original work (1974), the second-order diffraction involves only a special geometry in which both the receiver and the edge apex on the far side are in the shadow zone of the source, hence direct insonification and reflection can be completely ignored. To study the higher-order diffraction by edges with a more general geometry, we need to investigate the characteristics of the single diffraction by infinitely long straight wedge for some special cases. When the receiver is on or very close to either the shadow boundary or reflection boundary, i.e., either $\cos \nu\left(\theta+\theta_{0}\right)=\cos \nu \pi$ or $\cos \nu\left(\theta-\theta_{0}\right)=\cos \nu \pi$, then $M_{\nu}=0$. Using Eqs. (7a) and (7b), the diffracted wave reduces to

$$
p_{\mathrm{dif}} \rightarrow S \frac{e^{i k L}}{2 L}
$$

In other words, the diffracted field is approaching onehalf of the reflected wave. This is consistent with the results in the frequency domain given by Sandness et al. (1983) and in the time domain given by Svensson and Calamia (2006) and Calamia and Svensson (2007). A special case is that when the wedge angle $\theta_{w}$ also approaches $\pi$ (half space), both the numerator and the denominator of $M_{\nu}$ given by Eq. (6b) tend to zero. However, by applying the L'Hospital's rule, it is straightforward to show that $M_{\nu} \rightarrow 0$ as $\theta_{w} \rightarrow \pi$, and consequently, $A_{D} \rightarrow 1 / 2$ is finite but $D_{ \pm} \rightarrow 0$ by Eq. (5). This is exactly what is expected since for $\theta_{w} \rightarrow \pi$, the diffraction should approach zero.

Next, we examine the continuity of the total field when the observation point crosses the shadow or reflection boundary. Without loss of generality, we assume the source angle $\theta_{0} \leqslant \pi$ and allow the receiver angle $\theta$ to vary between 0 and $\theta_{w}$ as shown in Fig. 3. There are three regions.

Region I:

$$
\begin{aligned}
& p_{\text {tot }}=p_{\text {inc }}+p_{\text {ref }}+p_{\text {dif }}, \quad 0 \leqslant \theta \leqslant \pi-\theta_{0}, \\
& M_{\nu}\left(\theta-\theta_{0}\right) \leqslant 0, \quad M_{\nu}\left(\theta+\theta_{0}\right) \leqslant 0 .
\end{aligned}
$$

Region II:

$$
\begin{aligned}
& p_{\text {tot }}=p_{\text {inc }}+p_{\text {dif }}, \quad \pi-\theta_{0} \leqslant \theta \leqslant \pi+\theta_{0}, \\
& M_{\nu}\left(\theta-\theta_{0}\right) \leqslant 0, \quad M_{\nu}\left(\theta+\theta_{0}\right) \geqslant 0 .
\end{aligned}
$$

Region III (shadow region):

$$
\begin{aligned}
& p_{\text {tot }}=p_{\text {dif }}, \quad \pi+\theta_{0} \leqslant \theta \leqslant \theta_{w}, \\
& M_{\nu}\left(\theta-\theta_{0}\right) \geqslant 0, \quad M_{\nu}\left(\theta+\theta_{0}\right) \geqslant 0 .
\end{aligned}
$$

The total field under two limiting conditions will be analyzed: (1) Both source and receiver are in the far field; and (2) either source or receiver is on the apex of the wedge.

Far field.

(1) Reflection boundary: $\theta \rightarrow \pi-\theta_{0}$.

(a) $\theta_{-} \rightarrow \pi-\theta_{0}$ (Region I):

$$
\begin{aligned}
& M_{\nu}\left(\theta+\theta_{0}\right) \rightarrow 0_{-} \rightarrow D_{+}=-\frac{1}{2}, \\
& p_{\text {dif }}= S \frac{e^{i k L}}{L}\left\{-\frac{1}{2}-\left(\frac{e^{i \pi / 4}}{\sqrt{2}}\right)\right. \\
& \times \frac{\sin \nu \pi}{\sqrt{1-\cos \nu \pi \cos \nu\left(2 \theta_{0}-\pi\right)}} \\
&\left.\times A_{D}\left[\Gamma M_{\nu}\left(2 \theta_{0}-\pi\right)\right]\right\},
\end{aligned}
$$

$$
\begin{aligned}
p_{\mathrm{tot}}= & p_{\mathrm{inc}}+p_{\mathrm{ref}}+p_{\mathrm{dif}} \\
= & p_{\mathrm{inc}}+S \frac{e^{i k L}}{L}+S \frac{e^{i k L}}{L}\left\{-\frac{1}{2}-\left(\frac{e^{i \pi / 4}}{\sqrt{2}}\right)\right. \\
& \times \frac{\sin \nu \pi}{\sqrt{1-\cos \nu \pi \cos \nu\left(2 \theta_{0}-\pi\right)}} \\
& \left.\times A_{D}\left[\Gamma M_{\nu}\left(2 \theta_{0}-\pi\right)\right]\right\} \\
= & p_{\mathrm{inc}}+S \frac{e^{i k L}}{2 L}-S \frac{e^{i k L}}{L}\left(\frac{e^{i \pi / 4}}{\sqrt{2}}\right) \\
& \times \frac{\sin \nu \pi}{\sqrt{1-\cos \nu \pi \cos \nu\left(2 \theta_{0}-\pi\right)}} \\
& \times A_{D}\left[\Gamma M_{\nu}\left(2 \theta_{0}-\pi\right)\right] .
\end{aligned}
$$


(b) $\theta_{+} \rightarrow \pi-\theta_{0}$ (Region II):

$$
\begin{aligned}
M_{\nu}(\theta & \left.+\theta_{0}\right) \rightarrow 0_{+} \rightarrow D_{+}=\frac{1}{2}, \\
p_{\text {dif }}= & S \frac{e^{i k L}}{L}\left\{\frac{1}{2}-\left(\frac{e^{i \pi / 4}}{\sqrt{2}}\right)\right. \\
& \times \frac{\sin \nu \pi}{\sqrt{1-\cos \nu \pi \cos \nu\left(2 \theta_{0}-\pi\right)}} \\
& \left.\times A_{D}\left[\Gamma M_{\nu}\left(2 \theta_{0}-\pi\right)\right]\right\},
\end{aligned}
$$

$$
\begin{aligned}
p_{\text {tot }}= & p_{\text {inc }}+p_{\text {dif }} \\
= & p_{\text {inc }}+S \frac{e^{i k L}}{L}\left\{\frac{1}{2}-\left(\frac{e^{i \pi / 4}}{\sqrt{2}}\right)\right. \\
& \times \frac{\sin \nu \pi}{\sqrt{1-\cos \nu \pi \cos \nu\left(2 \theta_{0}-\pi\right)}} \\
& \left.\times A_{D}\left[\Gamma M_{\nu}\left(2 \theta_{0}-\pi\right)\right]\right\}=p_{\text {inc }}+S \frac{e^{i k L}}{2 L} \\
& -S \frac{e^{i k L}}{L}\left(\frac{e^{i \pi / 4}}{\sqrt{2}}\right) \frac{\sin \nu \pi}{\sqrt{1-\cos \nu \pi \cos \nu\left(2 \theta_{0}-\pi\right)}} \\
& \times A_{D}\left[\Gamma M_{\nu}\left(2 \theta_{0}-\pi\right)\right] .
\end{aligned}
$$

Equations (14b) and (14d) are exactly the same, confirming the continuity of the total field across the reflection boundary. The subscripts of $\theta_{ \pm}$and $0_{ \pm}$stand for approaching $\theta$ and 0 from above $(+)$ and below ( -$)$, respectively. It can be seen that when the receiver approaches the reflection boundary from below [Eq. (14a)], part of the diffracted field approaches one-half of the reflected field but changes sign to compensate for the disappearance of the reflected field when the receiver enters Region II [Eq. (14c)].

(2) Shadow boundary: $\theta \rightarrow \pi+\theta_{0}$.

(a) $\theta_{-} \rightarrow \pi+\theta_{0}$ (Region II):

$$
\begin{aligned}
& M_{\nu}\left(\theta-\theta_{0}\right) \rightarrow 0_{-} \rightarrow D_{-}=-\frac{1}{2}, \\
& p_{\text {dif }}= S \frac{e^{i k L}}{L}\left\{-\frac{1}{2}-\left(\frac{e^{i \pi / 4}}{\sqrt{2}}\right)\right. \\
& \times \frac{\sin \nu \pi}{\sqrt{1-\cos \nu \pi \cos \nu\left(2 \theta_{0}+\pi\right)}} \\
&\left.\times A_{D}\left[\Gamma M_{\nu}\left(2 \theta_{0}+\pi\right)\right]\right\}, \\
& p_{\text {tot }}= p_{\text {inc }}+p_{\text {dif }} \\
&= S \frac{e^{i k L}}{L}+S \frac{e^{i k L}}{L}\left\{-\frac{1}{2}-\left(\frac{e^{i \pi / 4}}{\sqrt{2}}\right)\right. \\
& \times \frac{\sin \nu \pi}{\sqrt{1-\cos \nu \pi \cos \nu\left(2 \theta_{0}+\pi\right)}}
\end{aligned}
$$

$$
\begin{aligned}
& \left.\times A_{D}\left[\Gamma M_{\nu}\left(2 \theta_{0}+\pi\right)\right]\right\} \\
= & S \frac{e^{i k L}}{2 L}-S \frac{e^{i k L}}{L}\left(\frac{e^{i \pi / 4}}{\sqrt{2}}\right) \\
& \times \frac{\sin \nu \pi}{\sqrt{1-\cos \nu \pi \cos \nu\left(2 \theta_{0}+\pi\right)}} \\
& \times A_{D}\left[\Gamma M_{\nu}\left(2 \theta_{0}+\pi\right)\right] .
\end{aligned}
$$

(b) $\theta_{+} \rightarrow \pi+\theta_{0}$ (Region III):

$$
\begin{aligned}
M_{\nu}(\theta & \left.-\theta_{0}\right) \rightarrow 0_{+} \rightarrow D_{-}=\frac{1}{2}, \\
p_{\text {dif }}= & S \frac{e^{i k L}}{L}\left\{\frac{1}{2}-\left(\frac{e^{i \pi / 4}}{\sqrt{2}}\right)\right. \\
& \times \frac{\sin \nu \pi}{\sqrt{1-\cos \nu \pi \cos \nu\left(2 \theta_{0}+\pi\right)}} \\
& \left.\times A_{D}\left[\Gamma M_{\nu}\left(2 \theta_{0}+\pi\right)\right]\right\}, \\
p_{\text {tot }}= & p_{\text {dif }}=S \frac{e^{i k L}}{2 L}-S \frac{e^{i k L}}{L}\left(\frac{e^{i \pi / 4}}{\sqrt{2}}\right) \\
& \times \frac{\sin \nu \pi}{\sqrt{1-\cos \nu \pi \cos \nu\left(2 \theta_{0}+\pi\right)}} A_{D}\left[\Gamma M_{\nu}\left(2 \theta_{0}+\pi\right)\right] .
\end{aligned}
$$

Again, we see that the total field is continuous across the shadow boundary.

Receiver on the apex (near field).

The results given by Eq. (10) and those studied previously are valid only for receiver being away from the apex of a wedge. To find the exact diffracted field at the apex, we need to reevaluate the integral given in Eq. (2a)-(2c) directly instead of using the asymptotic solution given by Eqs. (3)-(5) and (6a) $-(6 c)$. By setting $r=0$ in Eq. (2a) $-(2 c)$, we obtain

$$
\begin{aligned}
p_{\text {dif }} & =-S \frac{e^{i k R_{0}}}{R_{0}} \frac{\sin \nu \pi}{2 \theta_{w}} \int_{-\infty}^{\infty} \sum_{ \pm} F_{s}\left(s, \theta \pm \theta_{0}\right) d s \\
& =-S \frac{e^{i k R_{0}}}{R_{0}} \sum_{q=1}^{4} \frac{\sin \nu x_{q}}{2 \theta_{w}} \int_{-\infty}^{\infty} \frac{d s}{\cosh \nu s-\cos \nu x} \\
& =-S \frac{e^{i k R_{0}}}{R_{0}} \frac{1}{2 \pi} \sum_{q=1}^{4} \sin \nu x_{q} \int_{0}^{\infty} \frac{d u}{\cosh u-\cos \nu x} \\
& =-S \frac{e^{i k R_{0}}}{R_{0}} \frac{1}{2 \pi} \sum_{q=1}^{4} \sin \nu x_{q} I_{q},
\end{aligned}
$$

where $R_{0}=\left[r_{0}^{2}+\left(z-z_{0}\right)^{2}\right]^{1 / 2}$. The terms $x_{1}, x_{2}, x_{3}$, and $x_{4}$ correspond to $\pi+\theta+\theta_{0}, \pi+\theta-\theta_{0}, \pi-\theta+\theta_{0}$, and $\pi-\theta-\theta_{0}$, respectively. From the first to the second line in Eq. (17a), we have also used the relation (Pierce, 1981, p.489) 
TABLE I. Values of $\left[x_{q}\right]$ and diffraction in three diffraction regions shown in Fig. 3 computed using Eqs. (11)-(13), (19), and (20). In obtaining the results, we have assumed $\nu=\pi / \theta_{w}<1$ and $0 \leqslant \theta_{0} \leqslant \pi$ in our computations. Note that since $r=0, p_{\text {inc }}=p_{\text {ref }}=S e^{i k R_{0}} / R_{0}$, where $R_{0}=\left[r_{0}^{2}+\left(z-z_{0}\right)^{2}\right]^{1 / 2}$.

\begin{tabular}{|c|c|c|c|}
\hline & Region I & Region II & Region III \\
\hline Restrictions & $\begin{array}{l}\theta \geqslant 0 \\
\theta+\theta_{0} \leqslant \pi\end{array}$ & $\begin{array}{l}\theta+\theta_{0} \geqslant \pi \\
\theta-\theta_{0} \leqslant \pi \\
\pi+\theta_{0} \leqslant \theta_{w}\end{array}$ & $\theta \leqslant \theta_{w}$ \\
\hline$\left[x_{1}\right]$ & $\pi+\theta+\theta_{0}$ & $\pi+\theta+\theta_{0}$ & $\pi+\theta+\theta_{0}$ \\
\hline$\left[x_{2}\right]$ & $\pi+\theta-\theta_{0}$ & $\pi+\theta-\theta_{0}$ & $\pi+\theta-\theta_{0}$ \\
\hline$\left[x_{3}\right]$ & $\pi-\theta+\theta_{0}$ & $\pi-\theta+\theta_{0}$ & $\pi-\theta+\theta_{0}+2 \theta_{w}$ \\
\hline$\left[x_{4}\right]$ & $\pi-\theta-\theta_{0}$ & $\pi-\theta-\theta_{0}+2 \theta_{w}$ & $\pi-\theta-\theta_{0}+2 \theta_{w}$ \\
\hline$\sum_{q=1}^{4}\left[x_{q}\right]$ & $4 \pi$ & $4 \pi+2 \theta_{w}$ & $4 \pi+4 \theta_{w}$ \\
\hline$p_{\text {dif }}$ & $-S \frac{e^{i k R_{0}}}{R_{0}}\left(2-\frac{2 \pi}{\theta_{w}}\right)$ & $-S \frac{e^{i k R_{0}}}{R_{0}}\left(1-\frac{2 \pi}{\theta_{w}}\right)$ & $S \frac{e^{i k R_{0}}}{R_{0}}\left(\frac{2 \pi}{\theta_{w}}\right)$ \\
\hline$p_{\text {tot }}$ & $\begin{array}{l}p_{\mathrm{inc}}+p_{\mathrm{ref}}+p_{\mathrm{dif}} \\
=S \frac{e^{i k R_{0}}}{R_{0}}\left(\frac{2 \pi}{\theta_{w}}\right)\end{array}$ & $\begin{array}{l}p_{\text {inc }}+p_{\text {dif }} \\
=S \frac{e^{i k R_{0}}}{R_{0}}\left(\frac{2 \pi}{\theta_{w}}\right)\end{array}$ & $\begin{array}{l}p_{\mathrm{dif}} \\
=S \frac{e^{i k R_{0}}}{R_{0}}\left(\frac{2 \pi}{\theta_{w}}\right)\end{array}$ \\
\hline
\end{tabular}

$$
\begin{aligned}
\sin \nu \pi \sum_{ \pm} F_{s}\left(s, \theta \pm \theta_{0}\right) & =\sum_{q=1}^{4} \frac{\sin \nu x_{q}}{\cosh \nu s-\cos \nu x} \\
& =\sum_{q=1}^{4} \frac{\sin \nu x_{q}}{\cosh \nu s+\cos \left(\pi-\nu x_{q}\right)} .
\end{aligned}
$$

The analytical expression for $I_{q}$ is tabulated in Gradshteyn and Ryzhik (1980) (3.514.1),

$$
I_{q}=\frac{1}{\sin \nu x_{q}}\left(\pi-\nu\left[x_{q}\right]\right),
$$

where the quantity in the square brackets may be expressed as

$$
\begin{aligned}
& {\left[x_{q}\right]=2 \theta_{w}+x_{q}, \quad x_{q}<0,} \\
& {\left[x_{q}\right]=x_{q}, \quad 0 \leqslant x_{q} \leqslant 2 \theta_{w},} \\
& {\left[x_{q}\right]=x_{q}-2 \theta_{w}, \quad x_{q}>2 \theta_{w} .}
\end{aligned}
$$

The above-presented results are obtained by ensuring that the angle $\left[x_{q}\right]$ falls in the range of $0 \leqslant\left[x_{q}\right] \leqslant 2 \theta_{w}$, where $x_{q}$ could be any combination of $\pi \pm \theta \pm \theta_{0}$. Substituting Eq. (18) into Eq. (17a) leads to

$$
p_{\mathrm{dif}}=-S \frac{e^{i k R_{0}}}{R_{0}} \sum_{q=1}^{4} \frac{1}{2}\left(1-\left[x_{q}\right] / \theta_{w}\right) .
$$

The values of $\left[x_{q}\right]$ in the three regions (Fig. 3) are listed in Table I. The total field at the apex is amplified by a factor of $2 \pi / \theta_{w}$, which is consistent with that discussed by Pierce (1981, p. 480).

Receiver position moves from the apex to the far field.

For a receiver position that varies continuously between the apex and the far field, to our knowledge, there are no closed-form analytical solutions for a $\mathrm{cw}$ with satisfactory accuracy available for a general case of an arbitrary wedge angle. We therefore need to evaluate the integral, Eq.

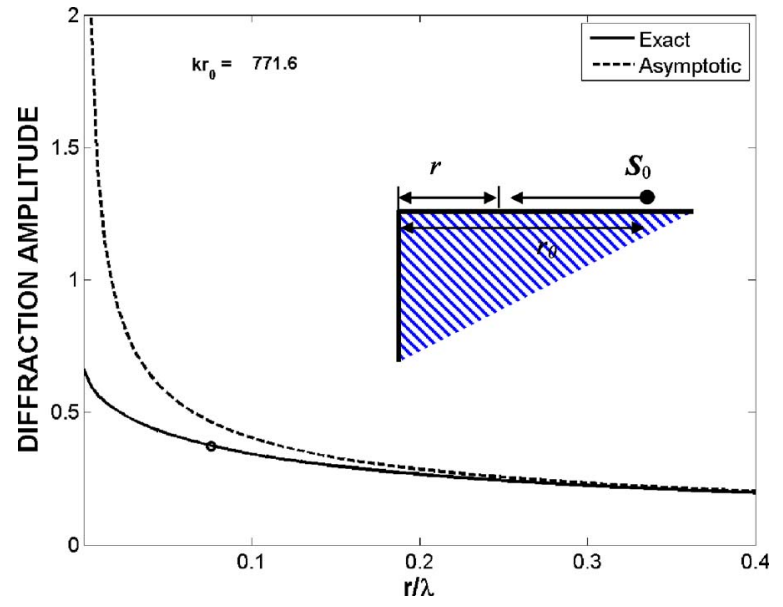

FIG. 4. (Color online) Comparison between the exact solution [Eq. (2a)-(2c) normalized by $\left.S e^{i k L} / L\right]$ and the diffraction amplitude defined in Eq. (5) and based on an asymptotic expansion of $D_{\Sigma}$, i.e., Eq. (9), as a function of range to the apex. The circle on the solid curve appearing at $r / \lambda \approx 0.08$ corresponds to the position about $1.9 \mathrm{~mm}$ away from the apex, or the thickness of the disk used in generating Fig. 2.

(2a)-(2c), numerically. For the case when the source is located on the surface of the wedge but in the far field region with the receiver location varying along the wedge surface from the wedge apex to the source, Fig. 4 illustrates the comparison between the exact and asymptotic solutions for a right-angle wedge $\left(\theta_{w}=3 \pi / 2\right)$. The solid curve is based on the exact integral solution, Eq. (2a)-(2c), computed numerically and normalized by $S e^{i k L} / L$ [see Eq. (3)], while the dashed curve is the diffraction amplitude, $D_{\Sigma}$, defined in Eq. (5) but using the asymptotic solution, Eq. (9). At $r / \lambda=0$, the value from the exact solution is $2 \pi / \theta_{w}=2 / 3$. The circle on the solid curve appearing at $r / \lambda \approx 0.08$ corresponds to the position of about $1.9 \mathrm{~mm}$ away from the apex, or the thickness of the disk used in generating Fig. 2.

\section{HIGHER-ORDER DIFFRACTION}

The diffraction by an infinitesimally thin edge, or a knife edge, can be characterized by first-order diffraction or single diffraction. However, real edges have a finite thickness and there may be higher-order diffraction associated with the boundaries of the "edge" (or, more precisely, truncated wedge) that have appreciable magnitude. In the early 1950s, Jones (1953) proposed an exact solution of the diffraction of electromagnetic waves by a thick semi-infinite plate for both parallel and perpendicular polarizations. The solutions involve contour integrals that cannot be easily evaluated. Keller and his colleagues presented approximate solutions for higher-order diffraction by an aperture on a hard screen, a problem that can be related to the scattering by a hard strip by applying Babinet's principle, using the method of the GTD when the width of the aperture is not too small (Keller, 1957, 1962; Karp and Keller, 1960). Medwin et al. (1982) used a discrete Huygens wavelet approach to describe the second-order diffraction by double edges. Tolstoy presented an exact solution to a truncated rigid wedge or a double edge (1989a,b), but his solution is derived based on a line source. Pierce (1974) used a ray-based "virtual source" approach and 


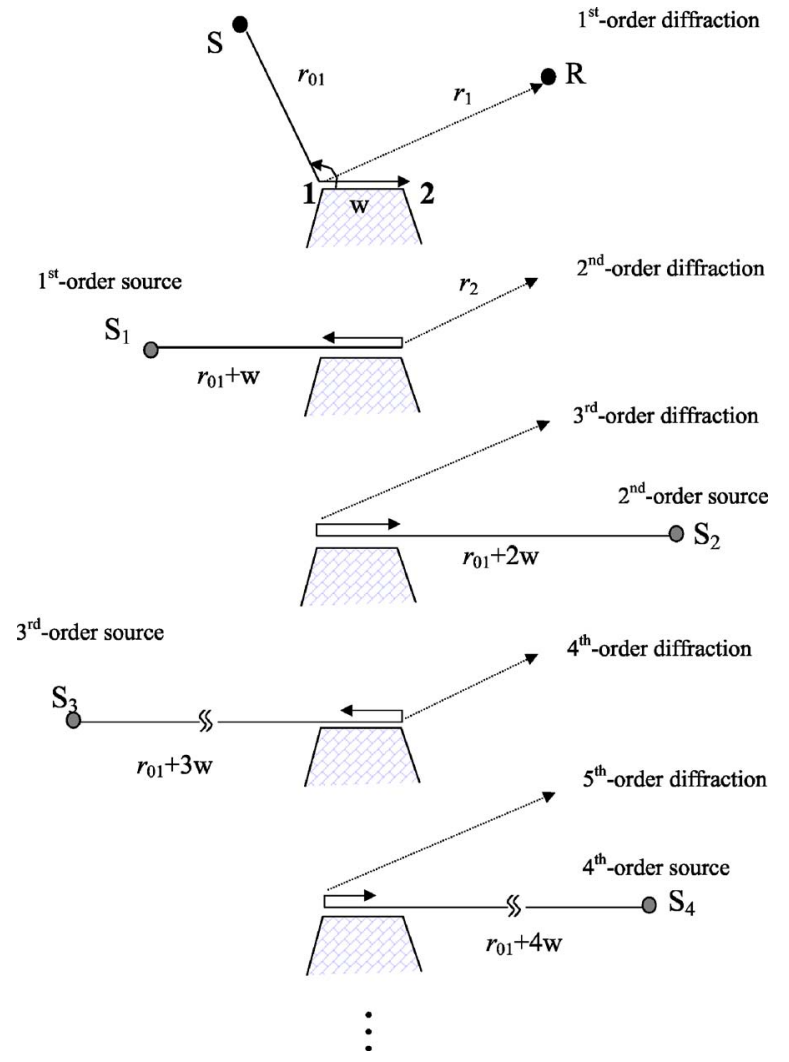

FIG. 5. (Color online) Diagram of higher-order diffraction.

presented an approximate solution of the second-order diffraction in a relatively simple and convenient analytical form.

Our approach is to follow Pierce's method and extend his second-order solution to include all orders, by requiring that the solution converges to the exact solution of an infinitely long knife edge as the thickness of the double edge approaches zero. The diagram of higher-order diffraction is depicted in Fig. 5. $S_{j}$ with $j=1,2, \ldots, \infty$ are the virtual sources located respectively at the hypothetical extension of the corresponding side of a wedge. The relationship between the consecutive virtual sources is assumed to be

$$
S_{j}=\alpha S_{j-1} D_{\Sigma_{p}},
$$

where $D_{\Sigma_{n}}$ is the first-order diffraction from the edge $p$, where $p=1$ or 2 corresponding to the edge number (Fig. 6), and $\alpha$ is a coefficient to be determined $(|\alpha|<1)$, which relates diffraction of order $j$ to that of order $j-1$. Note that $S_{0}$ is the source strength of the real source at the source position $S$ (Fig. 5). As discussed in Pierce (1974), once the width of the truncated wedge increases, the diffraction of order greater than two can be ignored and the "strength" of the virtual source approaches a value of one-half of that when the wedge is not extended because the total "effective" incident field is the sum of the virtual source and its image. It should be pointed out that since this method is based on the approximate representation, Eq. (3), that requires $k R \gg 1$, where $R$ is defined in Eq. (2c), it implies that the condition $k R \gg 1$ can still be satisfied even if $k r$ approaches zero provided that $k r_{0} \gg 1$.

Second-order diffraction.

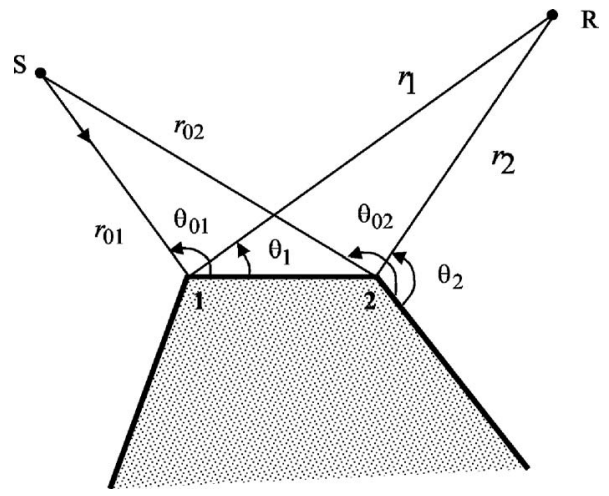

FIG. 6. Geometry illustrating model angles and ranges for the diffraction by an infinitely long straight truncated wedge or a double-edge. 2D crosssectional view.

There are two ray paths for the second-order diffraction that can be considered separately.

Path 1: $S \rightarrow 1 \rightarrow 2 \rightarrow R$

(a) $S \rightarrow 1 \rightarrow 2$ (first-order diffraction by edge "1" evaluated at edge 2 by assuming edge 2 is not present, i.e. both sides of edge 1 extend to infinity)

$$
p_{\mathrm{dif}}^{S 12}=S_{0} \frac{e^{i k\left(r_{01}+w\right)}}{r_{01}+w} D_{\Sigma_{1}}\left(w, 0 ; r_{01}, \theta_{01}\right) .
$$

(b) $S \rightarrow 1 \rightarrow 2 \rightarrow R$ (second-order diffraction by edge 2 evaluated at the receiver by assuming both sides of edge 2 extend to infinity)

$$
\begin{aligned}
p_{\mathrm{dif}}^{S 12 R} & =p_{\mathrm{dif}}^{S 12}\left(\frac{r_{01}+w}{r_{01}+w+r_{2}}\right) e^{i k r_{2}} D_{\Sigma_{2}}\left(r_{2}, \theta_{2} ; r_{01}+w, \theta_{w_{2}}\right) \\
& =\alpha S_{0} \frac{e^{i k L}}{L} D_{\Sigma_{1}}\left(w, 0 ; r_{01}, \theta_{01}\right) D_{\Sigma_{2}}\left(r_{2}, \theta_{2} ; r_{01}+w, \theta_{w_{2}}\right),
\end{aligned}
$$

where $L=r_{01}+w+r_{2}$.

Path 2: $S \rightarrow 2 \rightarrow 1 \rightarrow R$

(a) $S \rightarrow 2 \rightarrow 1$ (first-order diffraction by edge 2 evaluated at edge 1 by assuming both sides of edge 2 extend to infinity)

$$
p_{\mathrm{dif}}^{S 21}=S_{0} \frac{e^{i k\left(r_{02}+w\right)}}{r_{02}+w} D_{\Sigma_{2}}\left(w, \theta_{w} ; r_{02}, \theta_{02}\right) .
$$

(b) $S \rightarrow 2 \rightarrow 1 \rightarrow R$ (second-order diffraction by edge 1 evaluated at the receiver by assuming both sides of edge 1 extend to infinity)

$$
\begin{aligned}
p_{\mathrm{dif}}^{S 21 R} & =p_{\mathrm{dif}}^{S 21}\left(\frac{r_{02}+w}{r_{02}+w+r_{1}}\right) e^{i k r_{1}} D_{\Sigma_{1}}\left(r_{1}, \theta_{1} ; r_{02}+w, 0\right) \\
& =\alpha S_{0} \frac{e^{i k L}}{L} D_{\Sigma_{2}}\left(w, \theta_{w_{2}} ; r_{02}, \theta_{02}\right) D_{\Sigma_{1}}\left(r_{1}, \theta_{1} ; r_{02}+w, 0\right),
\end{aligned}
$$

where $L=r_{02}+w+r_{1}$.

If we set $\alpha=1 / 2$, and using the approximate solution of the diffraction function given in Eq. (9), the resultant solution for the second-order diffraction by a double edge is ex- 
actly the same as that given in Pierce (1974). Incorporating the same concept of virtual sources, we can extend the second-order solution to higher orders.

All orders of diffraction-General formulas.

Similar to the above-presented approach given for second-order diffraction, general formulas can be obtained for all orders of diffraction,

(1) Even Orders: $2(n+1)^{\text {th }}, n=0,1, \ldots$

$$
\begin{aligned}
\text { Path 1: } S \rightarrow 1 \rightarrow 2 \stackrel{\overbrace{\Leftrightarrow}^{n-1} 1}{\rightarrow} 1 \rightarrow 2 \rightarrow R \\
p_{\text {dif }}^{S 1(2 n 1) 2 R}=\alpha S_{0} \frac{e^{i k L_{n}}}{L_{n}} D_{\Sigma_{1}}\left(w, 0 ; r_{01}, \theta_{01}\right) D_{\Sigma_{2}}\left(r_{2}, \theta_{2} ; r_{01}\right. \\
\left.+(2 n+1) w, \theta_{w 2}\right)\left\{\prod _ { m = 0 } ^ { n - 1 } \alpha ^ { 2 } D _ { \Sigma _ { 2 } } \left(w, \theta_{w 2} ; r_{01}+(2 m\right.\right. \\
\left.\left.+1) w, \theta_{w 2}\right) D_{\Sigma_{1}}\left(w, 0 ; r_{01}+2(m+1) w, 0\right)\right\},
\end{aligned}
$$

where $L_{n}=r_{01}+(2 n+1) w+r_{2}$. In Eq. (24), the convention $\Pi_{m=0}^{-1}(\ldots) \equiv 1$ is assumed. Note that when $n=0$, Eq. (24) reduces to the second-order diffraction $p_{\mathrm{dif}}^{S 12 R}$, Eq. (22b).

$$
\begin{aligned}
\text { Path } 2: S & \rightarrow 2 \rightarrow \overbrace{p_{\mathrm{dif}}}^{S 2(1 n 2) 1 R}=\alpha S_{0} \frac{e^{i k L_{n}}}{L_{n}} D_{\Sigma_{2}}\left(w, \theta_{w 2} ; r_{02}, \theta_{02}\right) D_{\Sigma_{1}}\left(r_{1}, \theta_{1} ; r_{02}\right. \\
& +(2 n+1) w, 0)\left\{\prod _ { m = 0 } ^ { n - 1 } \alpha ^ { 2 } D _ { \Sigma _ { 1 } } \left(w, 0 ; r_{02}+(2 m\right.\right. \\
& \left.+1) w, 0) D_{\Sigma_{2}}\left(w, \theta_{w 2} ; r_{02}+2(m+1) w, \theta_{w 2}\right)\right\},
\end{aligned}
$$

where $L_{n}=r_{02}+(2 n+1) w+r_{1}$. Also note that when $n=0$, it reduces to the second-order diffraction $p_{\mathrm{dif}}^{S 21 R}$, Eq. (23b).
(2) Odd Orders: $(2 n+1)^{\text {th }}, n=1,2, \ldots$

Path 1: $S \rightarrow 1 \rightarrow 2 \overbrace{\Leftrightarrow}^{n-1} 1 \rightarrow R$.

$$
\begin{aligned}
p_{\mathrm{dif}}^{S 1(2 n 1) R}= & \alpha^{2} S_{0} \frac{e^{i k L_{n}}}{L_{n}} D_{\Sigma_{1}}\left(w, 0 ; r_{01}, \theta_{01}\right) D_{\Sigma_{2}}\left(w, \theta_{w 2} ; r_{01}\right. \\
& \left.+w, \theta_{w 2}\right) D_{\Sigma_{1}}\left(r_{1}, \theta_{1} ; r_{01}+2 n w, 0\right) \\
& \times\left\{\prod _ { m = 1 } ^ { n - 1 } \alpha ^ { 2 } D _ { \Sigma _ { 1 } } \left(w, 0 ; r_{01}\right.\right. \\
& \left.+2 m w, 0) D_{\Sigma_{2}}\left(w, \theta_{w 2} ; r_{01}+(2 m+1) w, \theta_{w 2}\right)\right\},
\end{aligned}
$$

where $L_{n}=r_{01}+2 n w+r_{1}$ and the convention $\Pi_{m=1}^{0}(\ldots) \equiv 1$ is assumed.

Path $2: S \rightarrow 2 \rightarrow 1 \overbrace{\Leftrightarrow}^{n-1} \rightarrow R$.

$$
\begin{aligned}
p_{\mathrm{dif}}^{S 2(1 n 2) R}= & \alpha^{2} S_{0} \frac{e^{i k L_{n}}}{L_{n}} D_{\Sigma_{2}}\left(w, \theta_{w 2} ; r_{02}, \theta_{02}\right) D_{\Sigma_{1}}\left(w, 0 ; r_{02}\right. \\
& +w, 0) D_{\Sigma_{2}}\left(r_{2}, \theta_{2} ; r_{02}+2 n w, \theta_{w 2}\right) \\
& \times\left\{\prod _ { m = 1 } ^ { n - 1 } \alpha ^ { 2 } D _ { \Sigma _ { 2 } } \left(w, \theta_{w 2} ; r_{02}\right.\right. \\
& \left.\left.+2 m w, \theta_{w 2}\right) D_{\Sigma_{1}}\left(w, 0 ; r_{02}+(2 m+1) w, 0\right)\right\},
\end{aligned}
$$

where $L_{n}=r_{02}+2 n w+r_{2}$.

Having obtained the diffraction for all even and odd orders, the total diffracted field will be the summation of all orders of diffraction including the first-order diffractions from both edges [see Fig. 1(a)_middle drawing]:

$$
p_{\text {dif,tot }}=\underbrace{p_{\mathrm{dif}}^{S 1 R}+p_{\mathrm{dif}}^{S 2 R}}_{\text {first-order }}+\overbrace{\underbrace{\infty}_{\text {higher-order }}}^{\sum_{n=0}^{\infty}\left(p_{\mathrm{dif}}^{S 1(2 n 1) 2 R}+p_{\mathrm{dif}}^{S 2(1 n 2) 1 R}\right)+\underbrace{\text { even }}_{\sum_{n=1}^{\infty}\left(p_{\mathrm{dif}}^{S 1(2 n 1) R}+p_{\mathrm{dif}}^{S 2(1 n 2) R}\right)} .}
$$

Note that even if $w \rightarrow 0$, the condition $k L_{n} \gg 1$ can still be satisfied as long as $k\left(r_{0 i}+r_{j}\right) \gg 1$, where $i, j=1,2$, and we can still use the approximate solution Eq. (3) to factor out the diffraction amplitude $D_{\Sigma}$ from the pressure field.

Determination of coefficent $\alpha$.
To determine the coefficient $\alpha$ included in Eq. (21), we need to apply the requirement that the total field $p_{\text {tot }}$ that includes the incident, reflected, and diffracted wave components is equal to $\left(2 \pi / \theta_{w}\right) e^{i k r_{0}} / r_{0}\left(r_{01}=r_{02}=r_{0}\right.$ as $\left.w \rightarrow 0\right)$ at the apex in the case of a knife edge or a double edge (Table I and also Pierce, 1981). The total diffracted fields in the different 
regions (Fig. 3), even as the observation point approaches to the apex $(w \rightarrow 0)$, should be consistent with those described by Eqs. (11)-(13). Each term in Eq. (28) can be expressed as a product of a source term, a propagation term, and a diffraction function, $S_{0}\left(e^{i k L} / L\right) D_{\Sigma}$, with $L=r+r_{0}$. Therefore, each summation term, in the limiting case of $w \rightarrow 0$, reduces to

$$
\begin{aligned}
& \sum_{n=0}^{\infty} p_{\mathrm{dif}}^{S 1(2 n 1) 2 R} \underset{w \rightarrow 0}{\longrightarrow} \alpha S_{0} D_{\Sigma_{1}}\left(0,0 ; r_{01}, \theta_{01}\right) D_{\Sigma_{2}}\left(r_{2}, \theta_{2} ; r_{01}, \theta_{w 2}\right) \\
& \quad \times \sum_{n=0}^{\infty} \alpha^{2 n}\left[D_{\Sigma_{2}}\left(0, \theta_{w 2} ; r_{01}, \theta_{w 2}\right) D_{\Sigma_{1}}\left(0,0 ; r_{01}, 0\right)\right]^{n} \\
& =\frac{\alpha D_{\Sigma_{1}}\left(0,0 ; r_{01}, \theta_{01}\right) D_{\Sigma_{2}}\left(r_{2}, \theta_{2} ; r_{01}, \theta_{w 2}\right)}{1-\alpha^{2} D_{\Sigma_{2}}\left(0, \theta_{w 2} ; r_{01}, \theta_{w 2}\right) D_{\Sigma_{1}}\left(0,0 ; r_{01}, 0\right)}
\end{aligned}
$$

where we have assumed $\mid \alpha^{2} D_{\Sigma_{2}}\left(0, \theta_{w 2} ; r_{01}, \theta_{w 2}\right)$ $\times D_{\Sigma_{1}}\left(0,0 ; r_{01}, 0\right) \mid<1$ to assure the convergence of the series summation in Eq. (29a). Similarly,

$$
\begin{aligned}
\sum_{n=0}^{\infty} p_{\mathrm{dif}}^{S 2(1 n 2) 1 R} \underset{w \rightarrow 0}{\longrightarrow} \alpha S_{0} D_{\Sigma_{2}}\left(0,0 ; r_{02}, \theta_{02}\right) D_{\Sigma_{1}}\left(r_{1}, \theta_{1} ; r_{02}, 0\right) \\
\quad \times \sum_{n=0}^{\infty} \alpha^{2 n}\left[D_{\Sigma_{1}}\left(0,0 ; r_{02}, 0\right) D_{\Sigma_{2}}\left(0, \theta_{w 2} ; r_{02}, \theta_{w 2}\right)\right]^{n} \\
=\frac{\alpha D_{\Sigma_{2}}\left(0, \theta_{w 2} ; r_{02}, \theta_{02}\right) D_{\Sigma_{1}}\left(r_{1}, \theta_{1} ; r_{02}, 0\right)}{1-\alpha^{2} D_{\Sigma_{1}}\left(0,0 ; r_{02}, 0\right) D_{\Sigma_{2}}\left(0, \theta_{w 2} ; r_{02}, \theta_{w 2}\right)}
\end{aligned}
$$

where, as in Eq. (29a), we have assumed $\left|\alpha^{2} D_{\Sigma_{1}}\left(0,0 ; r_{02}, 0\right) D_{\Sigma_{2}}\left(0, \theta_{w 2} ; r_{02}, \theta_{w 2}\right)\right|<1$ to assure the convergence of the series summation in Eq. (29b). The two summations for the odd orders are

$$
\begin{aligned}
\sum_{n=1}^{\infty} p_{\mathrm{dif}}^{S 1(2 n 1) R} \underset{w \rightarrow 0}{\longrightarrow} \alpha^{2} S_{0} D_{\Sigma_{1}}\left(0,0 ; r_{01}, \theta_{01}\right) D_{\Sigma_{2}}\left(0, \theta_{w 2} ; r_{01}, \theta_{w 2}\right) D_{\Sigma_{1}}\left(r_{1}, \theta_{1} ; r_{01}, 0\right) \\
\quad \times \sum_{n=0}^{\infty} \alpha^{2 n}\left[D_{\Sigma_{1}}\left(0,0 ; r_{01}, 0\right) D_{\Sigma_{2}}\left(0, \theta_{w 2} ; r_{01}, \theta_{w 2}\right)\right]^{n} \\
=\frac{\alpha^{2} D_{\Sigma_{1}}\left(0,0 ; r_{01}, \theta_{01}\right) D_{\Sigma_{2}}\left(0, \theta_{w 2} ; r_{01}, \theta_{w 2}\right) D_{\Sigma_{1}}\left(r_{1}, \theta_{1} ; r_{01}, 0\right)}{1-\alpha^{2} D_{\Sigma_{1}}\left(0,0 ; r_{01}, 0\right) D_{\Sigma_{2}}\left(0, \theta_{w 2} ; r_{01}, \theta_{w 2}\right)}
\end{aligned}
$$

and

$$
\begin{aligned}
& \sum_{n=0}^{\infty} p_{\mathrm{dif}}^{S 2(1 n 2) R} \underset{w \rightarrow 0}{\longrightarrow} \alpha^{2} S_{0} D_{\Sigma_{2}}\left(0, \theta_{w 2} ; r_{02}, \theta_{02}\right) D_{\Sigma_{1}}(0,0 ; \\
& \left.\quad \times r_{02}, 0\right) D_{\Sigma_{2}}\left(r_{2}, \theta_{2} ; r_{02}, \theta_{w 2}\right) \\
& \quad \times \sum_{n=0}^{\infty} \alpha^{2 n}\left[D_{\Sigma_{2}}\left(0, \theta_{w 2} ; r_{02}, \theta_{w 2}\right) D_{\Sigma_{1}}\left(0,0 ; r_{02}, 0\right)\right]^{n} \\
& =\frac{\alpha^{2} D_{\Sigma_{2}}\left(0, \theta_{w 2} ; r_{02}, \theta_{02}\right) D_{\Sigma_{1}}\left(0,0 ; r_{02}, 0\right) D_{\Sigma_{2}}\left(r_{2}, \theta_{2} ; r_{02}, \theta_{w 2}\right)}{1-\alpha^{2} D_{\Sigma_{2}}\left(0, \theta_{w 2} ; r_{02}, \theta_{w 2}\right) D_{\Sigma_{1}}\left(0,0 ; r_{02}, 0\right)},
\end{aligned}
$$

respectively. Since as $w \rightarrow 0, r_{01}=r_{02}=r_{0}, r_{1}=r_{2}=r$, and the double edge reduces to a single knife edge with a wedge angle:

$$
\theta_{s w}=\theta_{w 1}+\theta_{w 2}-\pi
$$

Note that for $w \rightarrow 0, L_{n}$ 's in Eqs. (24)-(27) are the same. Using Eq. (3), the total diffraction amplitude, $D_{\Sigma_{\theta_{s w}}}$, can be obtained by normalizing Eq. (28) with $S_{0} e^{i k L} / L$ and can be written as

$$
\begin{aligned}
D_{\Sigma_{\theta_{s w}}}\left(r, \theta ; r_{0}, \theta_{0}\right)= & D_{\Sigma_{\theta_{w 1}}}\left(r, \theta+\pi-\theta_{w 2} ; r_{0}, \theta_{0}+\pi-\theta_{w 2}\right) \\
& +D_{\Sigma_{\theta_{w 2}}}\left(r, \theta ; r_{0}, \theta_{0}\right)+D_{\Sigma_{m}}(\alpha)
\end{aligned}
$$

where the last term, $D_{\Sigma_{m}}(\alpha)$, on the right-hand side of Eq.
(31) represents the summation of four multiple-order diffractions given by Eqs. (29a)-(29d). In all of the abovepresented equations, Eqs. (21), (22a), (22b), (23a), (23b), (24)-(28), (29a)-(29d), (30), and (31), the angles are measured counterclockwise from the right side of the corresponding wedge (Fig. 6). In addition, we have assumed that $r \gg w$ and $r_{0} \gg w$, which lead to $\theta_{01}=\theta_{0}+\pi-\theta_{w 2}$ and $\theta_{1}=\theta$ $+\pi-\theta_{w 2}$. Solving Eq. (31) for an angle dependent $\alpha(\theta)$, we obtain a coefficient that characterizes the virtual source, $\alpha_{0}(\theta)$, for $w=0$. On the other hand, we know that when $w$ $\rightarrow \infty, \alpha_{\infty}(\theta) \rightarrow 1 / 2$ as discussed previously in Sec. III B (see also Pierce, 1974). To obtain $\alpha$ for an arbitrary $w$, we use an interpolation function 


$$
\alpha_{w}(\theta)=\left[\alpha_{0}(\theta)-\alpha_{\infty}(\theta)\right] e^{-\beta w / \lambda}+\alpha_{\infty}(\theta),
$$

where $\lambda$ is the wavelength and the coefficient $\beta$ controls the rate of $\alpha_{w}(\theta)$ approaching $\alpha_{\infty}(\theta)$. This function, chosen heuristically, is used because it smoothly connects the limiting values of $\alpha$ over the full range $0 \leqslant w<\infty$. The coefficient $\beta$ can be determined through comparison of the prediction with either data (which will be the case in this paper) or with an exact solution.

Having obtained $\alpha_{w}(\theta)$, we can compute all diffraction terms defined in Eqs. (24)-(27), and hence the total diffraction given in Eq. (28). It should be pointed out that Eq. (28) is based on the scattering geometry shown in Fig. 6. If, however, one of the edges is in the shadow zone of the source, such as edge 2 is in the shadow zone or $\theta_{01}>\pi$, the corresponding first-order diffraction term, $p_{\mathrm{dif}}^{S 2 R}$, as well as the corresponding terms for the higher-order diffraction, $p_{\text {dif }}^{S 2(1 n 2) 1 R}$ and $p_{\text {dif }}^{S 2(1 n 2) R}$, should be removed. Furthermore, if the receiver is also in the shadow zone relative to one of the edges, such as $\theta_{2}<\theta_{w 2}-\pi$, which is the geometry studied in Pierce (1974), the remaining first-order diffraction term, $p_{\text {dif }}^{S 1 R}$, and an additional higher-order diffraction term, $p_{\mathrm{dif}}^{S 1(2 n 1) R}$, should also be removed. As a result, the total diffraction contains only one term of the higher-order diffraction, $p_{\mathrm{dif}}^{S 1(2 n 1) 2 R}$.

\section{RESULTS AND DISCUSSION}

Numerical example: Determination of $\alpha(\theta)$ for a semiinfinite plate of finite thickness.

For a plate, we have $\theta_{w 1}=\theta_{w 2}=\theta_{w}=3 \pi / 2$, which results in $D_{\Sigma_{1}}=D_{\Sigma_{2}}=D_{\Sigma}$ and $\theta_{s w}=2 \pi$ by Eq. (30). Furthermore, if we assume a backscattering geometry and let $w \rightarrow 0$, we have $\theta_{2}=\theta_{02}=\theta, \theta_{1}=\theta_{01}=\theta-\pi / 2$ (Fig. 6), and $r_{01}=r_{02}=r_{0}=r_{1}$ $=r_{2}=r$. Using the symmetry of the wedge, we have

$$
\begin{aligned}
D_{\Sigma_{2}}\left(0, \theta_{w 2} ; r_{01}, \theta_{w 2}\right) & =D_{\Sigma_{1}}\left(0,0 ; r_{01}, 0\right)=D_{\Sigma_{1}}\left(0,0 ; r_{02}, 0\right) \\
& =D_{\Sigma_{2}}\left(0, \theta_{w 2} ; r_{02}, \theta_{w 2}\right) \\
& =D_{\Sigma}\left(0,0 ; r_{0}, 0\right)=D_{\Sigma}(0,0 ; r, 0) .
\end{aligned}
$$

Thus, the denominators in Eqs. (29a)-(29d) can be expressed as

$$
\text { Denominator }=1-\alpha^{2} D_{\Sigma}^{2}(0,0 ; r, 0) \text {. }
$$

By combining Eq. (29a) with Eq. (29d), and Eq. (29b) with Eq. (29c), the summation of the four terms involving higher-order diffraction in Eq. (31) can be rewritten as

$$
\begin{aligned}
D_{\Sigma_{m}}(\alpha)= & \frac{\alpha D_{\Sigma}\left(r, \theta ; r, \theta_{w}\right)\left[D_{\Sigma}(0,0 ; r, \theta-\pi / 2)+\alpha D_{\Sigma}\left(0, \theta_{w} ; r, \theta\right) D_{\Sigma}(0,0 ; r, 0)\right]}{1-\alpha^{2} D_{\Sigma}^{2}(0,0 ; r, 0)} \\
& +\frac{\alpha D_{\Sigma}(r, \theta-\pi / 2 ; r, 0)\left[D_{\Sigma}\left(0, \theta_{w} ; r, \theta\right)+\alpha D_{\Sigma}(0,0 ; r, \theta-\pi / 2) D_{\Sigma}(0,0 ; r, 0)\right]}{1-\alpha^{2} D_{\Sigma}^{2}(0,0 ; r, 0)} \\
= & \frac{\alpha D_{\Sigma}\left(0, \theta_{w} ; r, \theta\right)\left[D_{\Sigma}\left(r, \theta ; r, \theta_{w}\right)+D_{\Sigma}(r, \theta-\pi / 2 ; r, 0)\right]}{1-\alpha D_{\Sigma}(0,0 ; r, 0)} .
\end{aligned}
$$

To obtain the last result of Eq. (35) we limited the scattering angle by $\pi<\theta<3 \pi / 2$, a condition consistent with the experiment configuration discussed in the next section. As a result, $D_{\Sigma}(0,0 ; r, \theta-\pi / 2), D_{\Sigma}\left(0, \theta_{w} ; r, \theta\right)$, and $D_{\Sigma}(0,0 ; r, 0)$ are all in Region I (Fig. 3), and thus have the same value (Table I):

$$
\begin{aligned}
D_{\Sigma}\left(0, \theta_{w} ; r, \theta\right) & =D_{\Sigma}(0,0 ; r, \theta-\pi / 2) \\
& =D_{\Sigma}(0,0 ; r, 0)=\frac{2 \pi}{\theta_{w}}-2=-2 / 3 .
\end{aligned}
$$

Substituting Eq. (36) into Eq. (35) leads to

$$
D_{\Sigma_{m}}(\alpha)=-\left(\frac{2 \alpha}{3}\right) \frac{\left[D_{\Sigma}\left(r, \theta ; r, \theta_{w}\right)+D_{\Sigma}(r, \theta-\pi / 2 ; r, 0)\right]}{1+2 \alpha / 3} .
$$

If we now express $\theta_{D}$ in terms of $\theta_{w}$ and $\theta$ as illustrated in Fig. 2,

$$
\theta_{D}=\theta_{w}-\theta,
$$

we are able to apply the simulation results directly to the diffraction by the leading edge of a circular disk. For $\pi<\theta$ $<3 \pi / 2$, we have $0<\theta_{D}<\pi / 2$. Inserting Eq. (37) into Eq. (31) and replacing $\theta$ with $\theta_{D}$ result in

$$
\begin{aligned}
& D_{\Sigma_{2 \pi}}\left(r, 3 \pi / 2-\theta_{D} ; r, 3 \pi / 2-\theta_{D}\right)=D_{\Sigma_{3 \pi / 2}}\left(r, \pi-\theta_{D} ; r, \pi-\theta_{D}\right) \\
& \quad+D_{\Sigma_{3 \pi / 2}}\left(r, 3 \pi / 2-\theta_{D} ; r, 3 \pi / 2-\theta_{D}\right)-\left(\frac{2 \alpha}{3}\right) \\
& \quad \times \frac{D_{\Sigma_{3 \pi / 2}}\left(r, 3 \pi / 2-\theta_{D} ; r, \theta_{w}\right)+D_{\Sigma_{3 \pi / 2}}\left(r, \pi-\theta_{D} ; r, 0\right)}{1+2 \alpha / 3} .
\end{aligned}
$$

In Eq. (39), we use subscripts $2 \pi$ and $3 \pi / 2$ to differentiate the wedge angles between a knife edge $\left(\theta_{w}=2 \pi\right)$ and a right-angle wedge $\left(\theta_{w}=3 \pi / 2\right)$ explicitly. If we let

$$
D_{\Sigma_{\text {knife }}}=D_{\Sigma_{2 \pi}}\left(r, 3 \pi / 2-\theta_{D} ; r, 3 \pi / 2-\theta_{D}\right),
$$




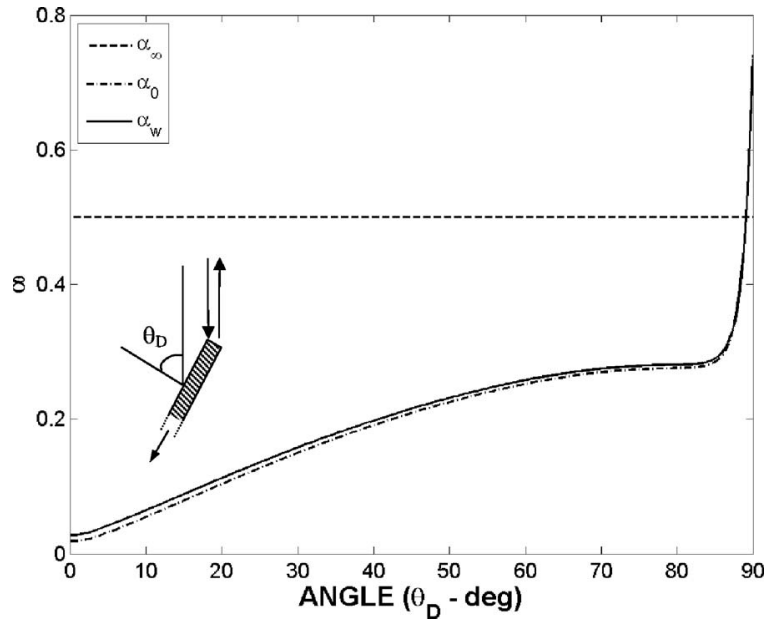

FIG. 7. Coefficient $\alpha$ vs angle for several cases. The dashed curve corresponds to the limit of the thickness $w \rightarrow \infty$, the dot-dashed curve is for the limit $w \rightarrow 0$ determined by Eq. (41), and the solid curve corresponds to interpolated $\alpha$ computed from the interpolation function Eq. (32) with $w$ $=1.9 \mathrm{~mm}$ and the frequency of $60 \mathrm{kHz}$ and a fit to laboratory data, as described later in this section.

$$
\begin{aligned}
D_{\Sigma_{1 \mathrm{st}}}= & D_{\Sigma_{3 \pi / 2}}\left(r, \pi-\theta_{D} ; r, \pi-\theta_{D}\right)+D_{\Sigma_{3 \pi / 2}}(r, 3 \pi / 2 \\
& \left.-\theta_{D} ; r, 3 \pi / 2-\theta_{D}\right),
\end{aligned}
$$

and

$$
D_{\Sigma_{\text {high }}}=D_{\Sigma_{3 \pi / 2}}\left(r, 3 \pi / 2-\theta_{D} ; r, \theta_{w}\right)+D_{\Sigma_{3 \pi / 2}}\left(r, \pi-\theta_{D} ; r, 0\right)
$$

represent the terms associated with the diffraction by the single knife edge, the first-order diffraction by the double edge, and the higher-order diffraction by the double edge, the coefficient $\alpha_{0}\left(\theta_{D}\right)$ can be obtained by

$$
\alpha_{0}\left(\theta_{D}\right)=-\frac{3}{2}\left(1+\frac{D_{\Sigma_{\text {high }}}}{D_{\Sigma_{\text {knife }}}-D_{\Sigma_{1 \mathrm{st}}}}\right)^{-1} .
$$

The computed coefficient $\alpha_{0}\left(\theta_{D}\right)$ (dot-dashed line) is illustrated in Fig. 7. From Fig. 7, we see that $\alpha_{0}\left(\theta_{D}\right)$ is less than $\frac{1}{2}$ when $\theta_{D}$ is well away from edge-on incidence, or $\theta_{D}<<\pi / 2$, indicating a small contribution from the higherorder diffraction to the total diffraction field. As $\theta_{D}$ increases but is not too close to edge-on incidence, $\alpha_{0}\left(\theta_{D}\right)$ increases with a moderate rate (slope). Once the scattering angle $\theta_{D}$ approaches the edge-on angular position, $\theta_{D}>85^{\circ}$, the coefficient $\alpha_{0}\left(\theta_{D}\right)$ increases with a much higher rate and approaches the value of $\frac{3}{4}$ at the edge-on angular position $\left(\theta_{D}\right.$ $=\pi / 2$ ), indicating a much increased contribution from the higher-order diffraction.

One of the interesting problems in edge diffraction we want to address is how thin a plate needs to be in order for a plate to behave as a knife edge, or a screen, for which only the first-order diffraction from a knife edge $\left(\theta_{w}=2 \pi\right)$ needs to be considered. Jones (1953) suggested that for the case of a plane incident wave, once the thickness of the plate is one-tenth of the acoustic wavelength, the plate can be treated as a knife edge. However, Jones did not provide any angle dependence or a quantitative basis for the statement. An example of such a problem is illustrated in Fig. 8, where the

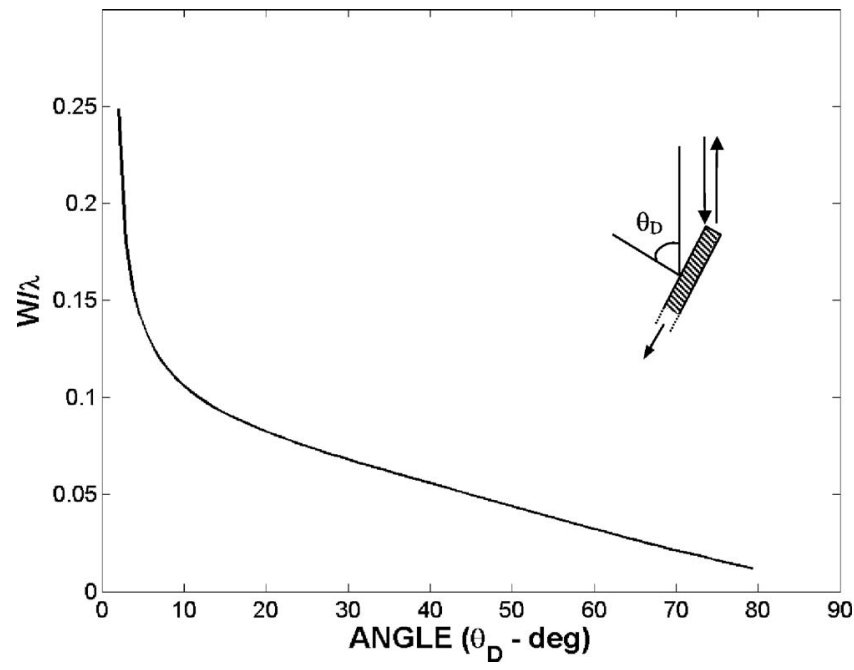

FIG. 8. The ratio of width to wavelength of the truncated wedge, which is required to make the difference in edge diffraction between a knife edge and a double edge of finite thickness less than $5 \%$, vs the scattering angle for a backscattering geometry.

ratio of the plate width to the wavelength $(w / \lambda)$ that is required to provide a difference in diffraction level between a knife edge and a double edge of less than $5 \%$, or $0.5 \mathrm{~dB}$, is plotted as a function of $\theta_{D}$. It can be seen that at a scattering angle of $\theta_{D}<10^{\circ}, w / \lambda$ is greater than 0.1 , indicating that when the incident wave approaches the direction parallel to the normal of the plate flat surface (broadside incidence), the higher-order diffraction resulting from the interaction between the two edges can be ignored. As the scattering angle increases, the ratio or the projected width of the plate ( $D \cos \theta_{D}$, where $D$ is the diameter of the disk) decreases, indicating that more and more contribution from the higherorder diffraction has to be considered. For $\theta_{D}<2^{\circ}$, the difference between the knife-edge solution and Eq. (28) is less than $5 \%$. Further calculation indicates that for the limiting case as $\theta_{D} \rightarrow 0$, the maximum difference between the two models is less than $1.5 \%$.

When the scattering angle is greater than $80^{\circ}$, the ratio approaches zero, corresponding to a sudden change in slope of the $\alpha$ curve shown in Fig. 7. The physical explanation for this phenomenon is not clear at this point, it may be related to our heuristic approach that forces the total diffraction based on the ray representation, Eq. (28), to converge to the first-order diffraction by a knife edge once the width of a double edge approaches zero.

Figure 9 demonstrates the relative importance of the contributions from higher-order diffraction in a different way at two incident angles, $10^{\circ}$ and $89^{\circ}$, respectively. It can be seen that for an incident angle close to the broadside of the disk $\left(\theta_{D}=10^{\circ}\right)$, the total field including only the first-order diffraction can provide satisfactory result with a relative error of about $10 \%$ or less. In contrast, as the angle of incidence moves away from the broadside $\left(\theta_{D}=89^{\circ}\right)$, the relative error increases to more than $400 \%$ when including only the first-order diffraction, to about $70 \%$ when the secondorder diffraction is included, and to less than $10 \%$ when the third-order diffractions are included. For the diffraction order 


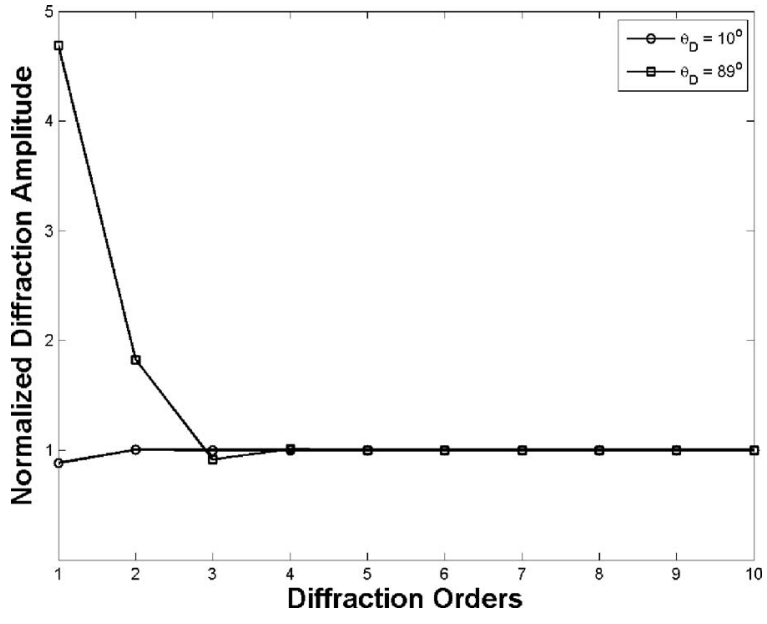

FIG. 9. Diffraction amplitude normalized by the converged amplitude ( $n$ $\rightarrow \infty$ ) as a function of diffraction orders at two different incident angles for a monostatic scattering geometry.

greater than 4 , the diffraction is basically indistinguishable from the converged value for $n \rightarrow \infty$. These results are consistent with those shown in Figs. 7 and 8.

Comparison with experimental data.

(1) Diffraction by metallic disks. Laboratory experiments involving a wide range of metal circular disks were conducted in Fall 2002. The purpose of the experiments was to understand the acoustic scattering by the seafloor covered with benthic shells. Metal disks were chosen as part of these free-field scattering measurements as they possess similar characteristics to the shells (Stanton and Chu, 2004). The experiments were conducted in an elongated rectangular tank with the scattering targets located about $3 \mathrm{~m}$ away from the transmitter-receiver pair in a near-backscattering configuration. Details of the experimental setup can be found in Stanton and Chu (2004). The PWTS, representing the edge diffraction from leading edge, was extracted from the backscattered time series by using a pulse compression technique (Chu and Stanton, 1998). Although the elasticity of the metal disk could also play a role in the diffraction process, the exclusion of the arrivals later than the first arrival for each ping can greatly reduce, if not eliminate, the influence resulting from the possible elastic surface and plate waves. In addition, since the reflection coefficient for an infinite aluminum plane is close to unity, the diffraction model based on the rigid wedge approximation is assumed to be valid in the following data/model comparison.

As mentioned in Sec. I, there are two aspects in constructing a complete diffraction model by a circular disk from the solution by an infinitely long knife edge. One is to take into account the finite thickness by including the higherorder diffraction, which is the subject of this paper, and the other is to account for the deformation of the edge. The deformation factor can be computed by a line integral over the leading edge of the disk and can be expressed as (Stanton et al., 2007):

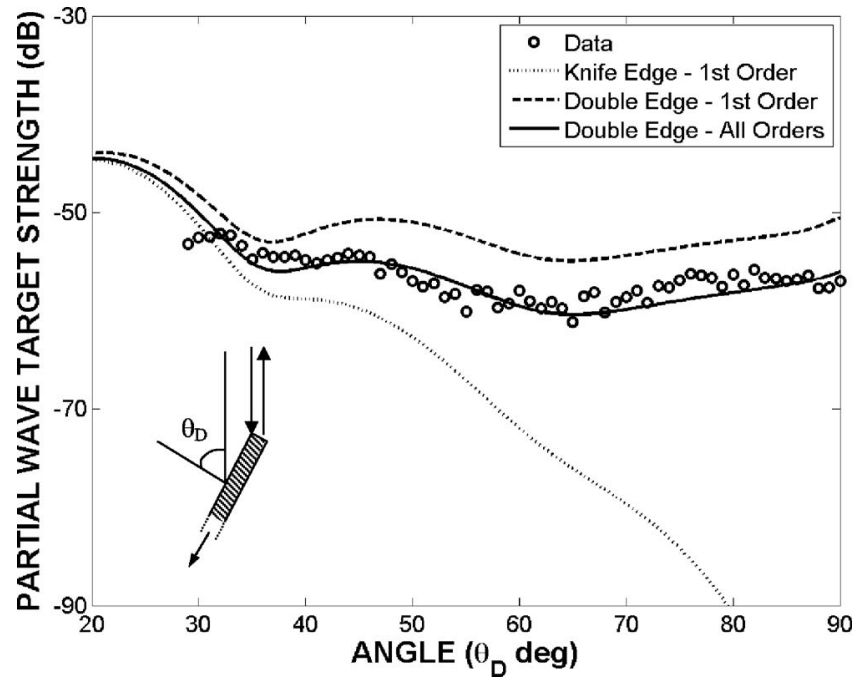

FIG. 10. Comparison of laboratory data $(60 \mathrm{kHz})$ involving an aluminum disk with various diffraction models

$$
f_{D}=a\left[J_{0}\left(2 k a \sin \theta_{D}\right)-i \frac{2}{\pi} s_{0,0}\left(2 k a \sin \theta_{D}\right)\right],
$$

where $a$ is the radius of the disk, $J_{0}(x)$ is the Bessel function of order zero, and $s_{0,0}$ is the Lommel function of order zero (Abramowitz and Stegun, 1974). The complete solution for the diffraction by a circular disk of finite thickness is the product of $p_{\text {dif,tot }}$ from Eq. (28) and the deformation factor $f_{D}$

A comparison of the model and the data is given in Fig. 10. The diffraction data as a function of backscattering angle shown in Fig. 2 are replotted in Fig. 10 (open circles), superimposed with several diffraction models. The disk is made of aluminum with a diameter of $8 \mathrm{~cm}$ and a thickness of about $1.9 \mathrm{~mm}(0.075 \mathrm{in}$.), which corresponds to $0.08 \lambda$ at $60 \mathrm{kHz}$ (open circle in Fig. 4). One of the diffraction models assumes a knife-edge geometry, or a semi-infinite screen (dotted). As described in Sec. I, the diffraction by a knifeedge decreases rapidly as $\theta_{D} \rightarrow \pi / 2$, and approaches zero once the incidence is edge-on. The contribution from the two first-order diffractions from edges 1 and 2 defined in Eq. (28) is also plotted in Fig. 10 (dashed). The solid curve representing the total diffraction, including all orders of diffraction, is computed from Eq. (28) with source coefficient $\alpha_{w}\left(\theta_{D}\right)$ computed from Eq. (32) and illustrated in Fig. 7 (solid). The interpolation coefficient, $\beta$ in Eq. (32) is assumed to have a linear relation with the ratio parameter, $w / \lambda$,

$$
\beta=\beta_{1} w / \lambda+\beta_{0} .
$$

Equation (43) results in a quadratic dependence of $w / \lambda$ for the exponent of the first term of the interpolation function in Eq. (32). The two constants $\beta_{0}=-2.7$ and $\beta_{1}=34.9$ are determined based on the visual fit of the computed PWTS to the data in Fig. 10. From Fig. 7, we see that the interpolated $\alpha_{w}\left(\theta_{D}\right)$ used to provide the "best" fit to the data is much closer to $\alpha_{0}\left(\theta_{D}\right)$ than to the asymptotic $\alpha_{\infty}\left(\theta_{D}\right)$, which is $\frac{1}{2}$.

Figure 11 shows the comparison of the model with the diffraction data from a number of aluminum disks with the same diameter but different thickness and at different back- 


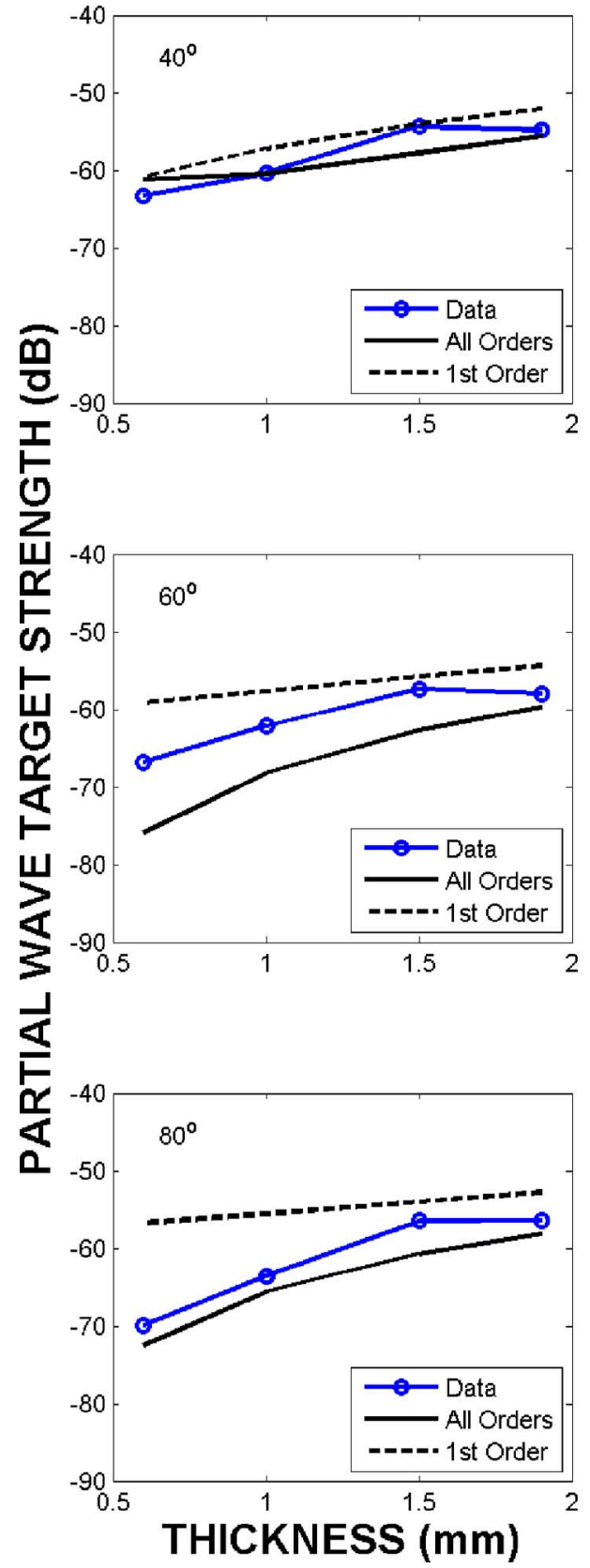

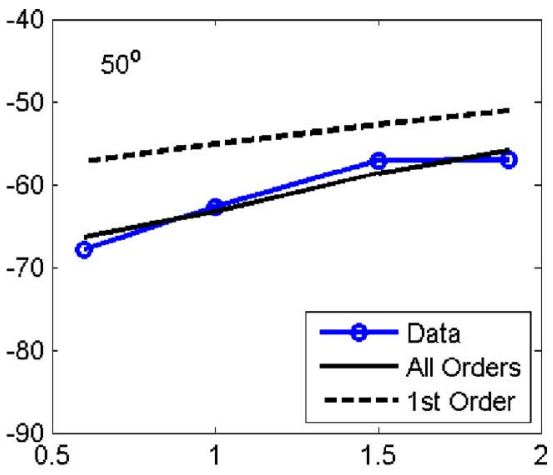

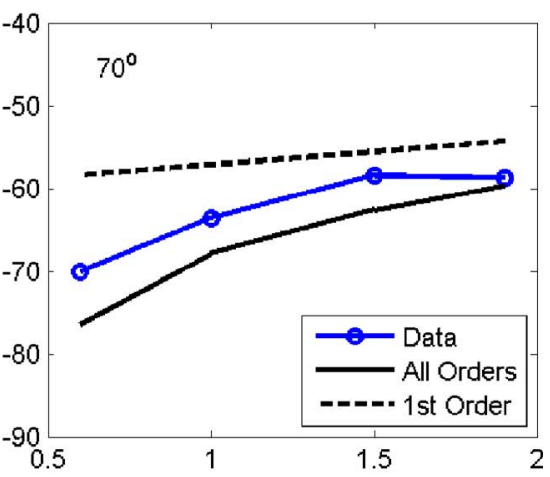

FIG. 11. (Color online) Comparison of the measured partial wave target strength of an aluminum disk (diameter of $8 \mathrm{~cm})$ of various thickness $(w$ $=0.6,1.0,1.5$, and $1.9 \mathrm{~mm}$ ) with the model of all orders of diffractions [Eq. (28)] at different angles. For the scattering angle at $90^{\circ}$ (bottom right), the actual angle is $89.6^{\circ}$ to exclude the "edge-on" specular term. scattering angles. The same value of $\beta_{0}$ and $\beta_{1}$ that were determined in Fig. 10 were used to compute the theoretical higher-order diffraction in each of the plots in Fig. 11. In general, the PWTS at all angles decreases as the disk becomes thinner. For all plots, the slopes of the model are very close to those of the data. The predicted absolute values of PWTS are in general agreement with some of the data but no more than $10 \mathrm{~dB}$ difference for the rest of the data such as at $60^{\circ}$. The comparison indicates that the proposed higher-order diffraction model has significant merit in describing the edge diffraction by circular disks of finite thickness. The mismatch in this comparison may result from a number of factors including approximations made in deriving the deformation factor, the assumptions made in deriving factor $\alpha$, and the approximation of neglecting the elasticity of the disks, as well as the uncertainty in measuring the scattering geometry. For the purpose of comparison, the model predictions includ- ing only the first-order diffraction are also presented, which overestimate the total diffraction in its overall level. Furthermore, the slope predicted by the double-edge first-order diffraction model approaches zero and deviates more and more from the data as $\theta_{D} \rightarrow 90^{\circ}$. This is consistent with the results of Figs. 9 and 10.

(2) Diffraction by a hard strip. Medwin et al. (1982) used the concept of Huygens impulsive wavelets to model the second-order diffraction by a hard strip. Their solution was based on the exact impulse solution of the diffraction by an infinitely long straight wedge (Biot and Tolstoy, 1957), or BT solution, and was transformed to the frequency domain. Their model predictions agreed with the experimental data very well. Here we will present an alternative solution to the problem to demonstrate the applicability of our approach.

For a hard strip, the $n$th order diffraction results from the contributions corresponding to $2^{n}$ different diffraction ray 


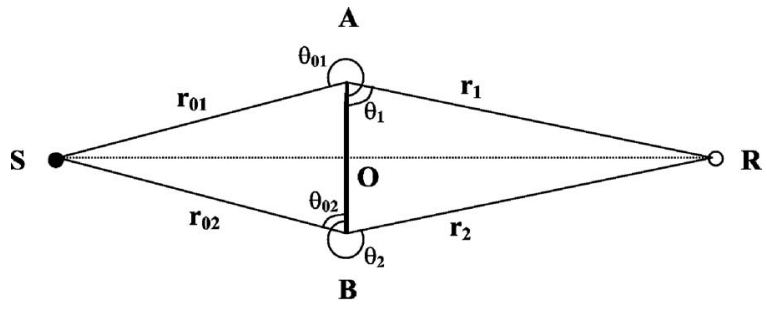

(a)

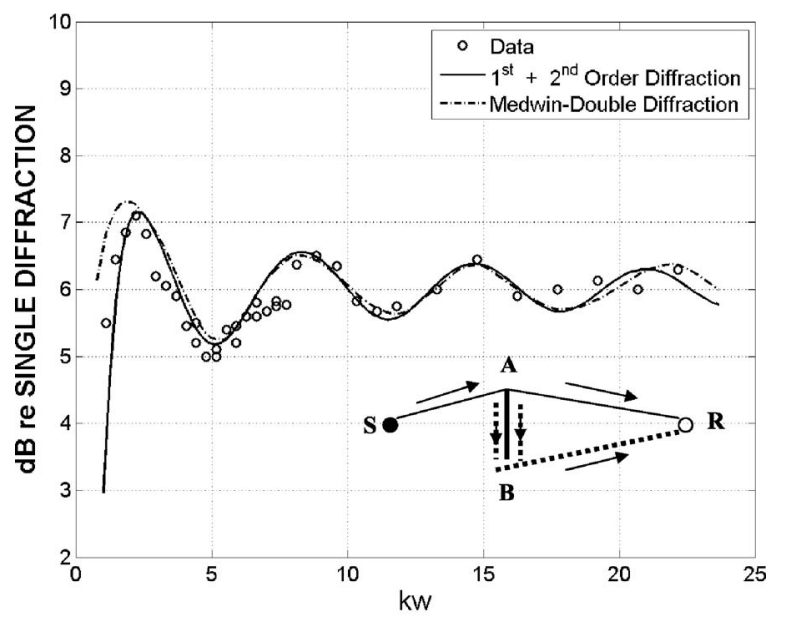

(b)

FIG. 12. (a) Geometry and notations of the parameters for diffraction by a hard strip. The view is along the infinite length of the strip and the distance between points " $A$ " and " $B$ " is the width $w$. (b) Comparison of data (Medwin et al., 1982) with the second-order diffraction model [Eq. (47)] (solid) and Medwin's "double" or second-order diffraction (dashed). The data are the ratio of the total field to that of one-half of the total first-order diffraction (ray paths $S A R$ and $S B R$ ). The model parameters are $\alpha_{0}=0.2, \alpha_{\infty}=0.5, \beta_{0}$ $=-2.7$, and $\beta_{1}=34.9$ [Eq. (32)]. The distances from the strip to the source and the receiver are $14.5 \mathrm{~cm}[O S$, see (a)] and $23.2 \mathrm{~cm}[O R$, see (a)], respectively. The width of the strip $(w)$ is $4 \mathrm{~cm}$. The frequencies are from about 1.5 to $30 \mathrm{kHz}$. Note that the horizontal axis in Medwin et al. is frequency in log-scale and we use a dimensionless variable $\mathrm{kw}$ on a linear scale, where $w$ is the width of the strip.

paths. This is because for each new higher-order diffraction there will be twice as many ray paths propagating along both sides of the strip. For this particular geometry, it is extremely hard, if not impossible, to find a general expression of the total diffraction in closed form by summing all orders of the diffraction similar to Eq. (28) with the explicit expressions given in Eqs. (24)-(27). As a result, we cannot use the same method to determine the magnitude of the virtual source, $\alpha_{0}$, as for the double-edge problem associated with a disk of finite thickness. However, since we know that if the value of $k w$, which can be determined from the width of the strip $(w)$ and the acoustic frequency used in their experiment, is large compared to unity, the diffraction with order higher than 2 is negligible. To compare directly with the laboratory experimental data for a hard strip published by Medwin et al. (1982), instead of deriving the solution for the total field as we did for the disk experiments, we now use the solutions given by Eq. (3), Eqs. (22a)-(22b), and Eqs. (23a)-(23b), to express as the ratio of the "total diffracted field" (first and second) to the first-order diffraction from edge " $k$," where $k$ can be either 1 or 2 corresponding to edge "A" and "B," respectively [Fig. 12(a)],

$$
\begin{aligned}
R_{N}= & +\frac{\alpha}{\xi_{k} D_{\Sigma_{k}}\left(r_{k}, \theta_{k} ; r_{0 k}, \theta_{0 k}\right)} \\
& \times\left\{\xi_{12} D_{\Sigma_{1}}\left(w, 0 ; r_{01}, \theta_{01}\right) D_{\Sigma_{2}}\left(r_{2}, \theta_{2} ; r_{01}+w, \theta_{w}\right)\right. \\
& +\xi_{12} D_{\Sigma_{1}}\left(w, \theta_{w} ; r_{01}, \theta_{01}\right) D_{\Sigma_{2}}\left(r_{2}, \theta_{2} ; r_{01}+w, 0\right) \\
& +\xi_{21} D_{\Sigma_{2}}\left(w, 0 ; r_{02}, \theta_{02}\right) D_{\Sigma_{1}}\left(r_{1}, \theta_{1} ; r_{02}+w, \theta_{w}\right) \\
& \left.+\xi_{21} D_{\Sigma_{2}}\left(w, \theta_{w} ; r_{02}, \theta_{02}\right) D_{\Sigma_{1}}\left(r_{1}, \theta_{1} ; r_{02}+w, 0\right)\right\},
\end{aligned}
$$

where the factors $\xi_{k}$, with $k=1$ or 2 , is the propagation term associated with edge $\mathrm{A}$ or $\mathrm{B}$, and $\xi_{i j}$, where $i, j=1,2$, are the propagation terms corresponding to the different ray paths between the first- and second-order diffractions,

$$
\xi_{k}=\frac{e^{i k\left(r_{0 k}+r_{k}\right)}}{r_{0 k}+r_{k}}, \quad \xi_{i j}=\frac{e^{i k\left(r_{0 i}+w+r_{j}\right)}}{r_{0 i}+w+r_{j}} .
$$

For a symmetrical scattering geometry shown in Fig. 12(a), we have $r_{01}=r_{02} \equiv r_{0}, r_{1}=r_{2} \equiv r, \theta_{w_{1}}=\theta_{w_{2}} \equiv \theta_{w}, \theta_{01}$ $=\theta_{w}-\theta_{02} \equiv \theta_{0}$, and $\theta_{2}=\theta_{w}-\theta_{1} \equiv \theta$. Since $\theta_{w}=2 \pi$ and $\nu$ $=\pi / \theta_{w}=1 / 2$ for a strip, it is easy to show that

$$
\begin{aligned}
D_{\Sigma_{1}}\left(r_{1}, \theta_{1} ; r_{01}, \theta_{01}\right) & =D_{\Sigma_{2}}\left(r_{2}, \theta_{2} ; r_{02}, \theta_{02}\right) \\
& \equiv D_{\Sigma}\left(r, \theta ; r_{0}, \theta_{w}-\theta_{0}\right) \\
& =-D_{\Sigma}\left(r, \theta ; r_{0}, \theta_{0}\right) \\
& =D_{\Sigma}\left(r, \theta_{w}-\theta ; r_{0}, \theta_{0}\right) .
\end{aligned}
$$

Using these relations, Eq. (44) can be simplified to

$$
\begin{aligned}
R_{N}= & -4 \alpha e^{i k w}\left(\frac{r+r_{0}}{r_{0}+r+w}\right) \\
& \times \frac{D_{\Sigma}\left(w, 0 ; r_{0}, \theta_{0}\right) D_{\Sigma}\left(r, \theta ; r_{0}+w, 0\right)}{D_{\Sigma}\left(r, \theta ; r_{0}, \theta_{0}\right)} .
\end{aligned}
$$

Comparison of the computed theoretical predictions with the laboratory experimental data published by Medwin et al. (1982) is shown in Fig. 12(b). Since the experiment was performed in the air, the strip could be regarded approximately as a "hard" material. The model parameters needed to compute $\alpha_{w}(\theta)$ in Eq. (32) are $\alpha_{0}=0.2$ and $\alpha_{\infty}=0.5$. As stated previously, the determination of $\alpha_{0}$ for this problem is different from the backscattering case [Eq. (39)]. As an approximation, we choose 0.2, an average value of $\alpha_{0}$ shown in Fig. 7 , as the estimated $\alpha_{0}$ value. The same values of $\beta_{0}$ and $\beta_{1}$ that were used in Fig. 10 with the disk data were also used in obtaining Fig. 12(b). The width of the strip, $w$, is $4 \mathrm{~cm}$. The frequencies vary from about 15 to $30 \mathrm{kHz}$. The distances from the strip to the source and the receiver are $14.5 \mathrm{~cm}[\overline{\mathrm{OS}}$, see Fig. 12(a)] and $23.2 \mathrm{~cm}$ [ $\overline{\mathrm{OR}}$, see Fig. 12(a)], respectively. The agreement between the data and the theory is very good over a wide range of $k w$. To illustrate the difference between Medwin's second-order diffraction model (Medwin et al., 1982) and the solution presented here, we superimposed the theoretical predictions from their diffraction model as a comparison. It is seen that at lower frequencies, or small $k w$, the present model provides better fit and the two models are converged as $k w$ increases. This is expected since at lower frequencies, higher-order diffraction is important (see 
Fig. 9). As $k w$ increases, $\alpha_{w}(\theta)$ approaches $\alpha_{\infty}=0.5$, and the current diffraction model reduces to the Pierce's secondorder diffraction model. Since the BT solution, from which Medwin's diffraction model was derived, is exactly the same as the Pierce's solution (see the Appendix), from which the current higher-order diffraction is derived, Medwin's secondorder diffraction model is essentially the same as our higherorder diffraction model for large $k w$. The deviation between the two theoretical models at higher frequencies $(k w>18)$ is likely due to the difficulties in numerical computations of the double diffraction.

From the three examples (Figs. 10-12), we can conclude that although the two empirical parameters, $\beta_{0}$ and $\beta_{1}$, are determined originally from fitting particular backscattering data set, the fact that the reasonable agreement between data and theory by using the same $\beta_{0}$ and $\beta_{1}$ for all three different cases, i.e., versus incident angle (Fig. 10), width (Fig. 11), and frequency (Fig. 12), suggests that the assumed relations given by Eqs. (32) and (43) work reasonably well at least for the diffraction by metal disks of finite thickness and by strip of finite width. Particularly, since the latter two examples involve varying $w / \lambda$, the reasonable agreement between the model and the data suggests that the approximation of the exponent term of the interpolation function, Eqs. (32) and (43), by a second-order polynomial of $w / \lambda$ in obtaining $\alpha$ may be justified. In addition, the three examples also suggest that the two parameters work well not only for backscattering application (Figs. 10 and 11 but also for bistatic scattering application (Fig. 12). This is actually expected since in theory, the general solution of Eq. (28), as well as Eq. (32), is valid for all scattering geometry.

\section{SUMMARY AND CONCLUSION}

We have developed an approximate analytical solution of bistatic scattering by a double edge of finite thickness that includes all orders of diffraction from the edges. The solution is an extension of a previously developed second-order diffraction model (Pierce, 1974). An amplitude function of the virtual source $(\alpha)$, a function of the scattering geometry, the thickness of the double edge, and the acoustic frequency, is introduced heuristically to make the ray-based solution converge to the exact solution of an infinitely long knife edge as the thickness of the double edge approaches zero. A heuristic interpolation function is used to obtain the $\alpha$ for a double edge of an arbitrary thickness with two empirically determined parameters.

The solution, when combined with the approximate solution to account for the deformation of a general edge (Stanton et al., 2007), is used to describe the edge diffraction by a disk of finite thickness. It is found that the contribution of the higher-order diffraction is small at or near broadside incidence, increases with the incident angle at a moderate rate if the incidence is not too close to edge-on, and increases drastically as the incidence approaches the edge-on direction $\left(\theta_{D} \rightarrow \pi / 2\right)$. The agreement between the theory and the data is generally good. An approximate and closed form solution of the diffraction by a hard strip that takes into account the first- and second-order diffractions is presented and is com- pared favorably with the laboratory experimental data over a wide range of $k w$ values, where $k$ is the wave number and $w$ is the width of the strip.

In addition, the equivalence between the exact impulse solution of an infinitely long straight wedge in the time domain (Biot and Tolstoy, 1957) and the integral solution in the frequency domain given by various authors (Sommerfeld, 1954; Pierce, 1981) has been shown. Futhermore, the behavior of the diffracted field due to the presence of an infinitely long straight wedge under different conditions has been studied systematically and analytically. It is found that the diffracted field increases drastically as it approaches either the reflection or shadow boundaries and has an amplitude value of one-half of the incident and/or reflected waves. At or near the apex of the wedge, the diffracted field is constant within the same diffraction regions but discontinuous across the reflection and shadow boundaries by a magnitude value of unity (Table I). It is also found that the edge diffraction has the same order of magnitude as the incident and/or the reflected fields.

Despite the promising comparison between the data and our higher-order diffraction model, it is recognized that in deriving the approximate solution for the diffraction by a double edge we have used several assumptions: (1) The relationship between the virtual sources of adjacent order specified in Eq. (21); (2) an empirical interpolation function defined by Eq. (32); and (3) a linear relation between the interpolation coefficient $\beta$ and $w / \lambda$ [Eq. (43)], where $w$ is the distance between the apexes of the two edges [Figs. 1(b) and 5] or the width of the strip [Fig. 12(a)] and $\lambda$ is the wavelength. These heuristic relations (assumptions) allow us to understand the more complicated double-edge diffraction problem involving all orders of diffraction in a relatively simple way and gain more physical insight into the mechanisms of the higher-order diffraction by a double edge.

In conclusion, we have derived an approximate raybased solution of diffraction by double edges in a general form that can take into account all orders of diffraction. The solution can, in principle, be extended to include more than two edges.

\section{ACKNOWLEDGMENTS}

The authors would like to thank the reviewers for their helpful comments and suggestions, which helped to improve the quality of the paper significantly. This work was supported by the US Office of Naval Research and by the Woods Hole Oceanographic Institution.

\section{APPENDIX: EQUIVALENCY BETWEEN THE EXACT TRANSIENT IMPULSE SOLUTION AND THE CW REPRESENTATION OF THE DIFFRACTED WAVE DUE TO AN INFINITELY LONG RIGID WEDGE}

Pierce (1981, pp. 489-490) briefly provided the procedures of how to convert the contour-integral-based spectral representation to a transient solution, but did not provide the closed-form impulse solution. Here we use a different method to prove the equivalency between the exact spectral representation [Eq. (2a)-(2c)] and the exact impulse solution given by Biot and Tolstoy (1957). 
The exact impulse solution of the diffracted wave due to a point source for a rigid wedge, given by Biot and Tolstoy (1957), is

$$
\begin{aligned}
p_{\text {diffr }}(t)= & \frac{\rho c}{4 \pi \theta_{w} r r_{0}} \frac{e^{-\nu \eta}}{\sinh \eta} \sum_{ \pm} \\
& \times \frac{\sin \left[\nu\left(\pi \pm \theta \pm \theta_{0}\right)\right]}{1-2 e^{-\nu \eta} \cos \left[\nu\left(\pi \pm \theta \pm \theta_{0}\right)\right]+e^{-2 \nu \eta}} u\left(t-\tau_{0}\right),
\end{aligned}
$$

where $u(x)$ is a unit step function and $\eta(t)$ is a function of time $t$ and the other geometric parameters

$$
\eta(t)=\cosh ^{-1} \frac{c^{2} t^{2}-\left(r^{2}+r_{0}^{2}+\left(z-z_{0}\right)^{2}\right)}{2 r r_{0}},
$$

where $c$ is the sound speed. $\tau_{0}$ is the shortest time required for the wave traveling from the source to the apex, and then to the receiver, or the time when $\eta(t)=0$,

$$
\tau_{0}=\frac{\left(\left(r+r_{0}\right)^{2}+\left(z-z_{0}\right)^{2}\right)^{1 / 2}}{c}=\frac{L}{c},
$$

where $L$ is given in Eq. (4). The source term $\rho$ is the volume flux and is related to the source strength $S$ in Eq. (2a)-(2c) by $S=-\rho / 4 \pi$, i.e., volume flux per unit solid angle. The summation in (A1) represents four combinations:,,+++--+ , and -- . It can be shown that straightforward manipulations of Eq. (A1) lead to

$$
p_{\text {diffr }}(t)=\frac{\rho c}{4 \pi \theta_{w} r r_{0}} \frac{\sin \nu \pi}{\sinh \eta} \sum_{ \pm} \frac{\cos \nu \pi-\cosh \nu \eta \cos \left[\nu\left(\theta \pm \theta_{0}\right)\right]}{\cos \nu \eta-\cos \left[\nu\left(\theta \pm \theta_{0}\right)\right]-\sin ^{2} \nu \eta \sin ^{2}\left[\nu\left(\theta \pm \theta_{0}\right)\right]} u\left(t-\tau_{0}\right)
$$

The spectral representation can be obtained via the Fourier transform of Eq. (A1) as

$$
p_{\mathrm{dif}}(f)=\int_{-\infty}^{\infty} p_{\mathrm{diffr}}(t) e^{i \omega t} d t=\int_{\tau_{0}}^{\infty} p_{\mathrm{diffr}}(t) e^{i \omega t} d t
$$

Using Eq. (A2), we can change the integration variable $t$ to $\eta$,

$$
p_{\text {dif }}(f)=\frac{\rho \sin \nu \pi}{4 \pi \theta_{w}} \int_{0}^{\infty} \frac{e^{i k R(\eta)}}{R(\eta)} \sum_{ \pm} \frac{\cos \nu \pi-\cosh \nu \eta \cos \left[\nu\left(\theta \pm \theta_{0}\right)\right]}{\cos \nu \eta-\cos \left[\nu\left(\theta \pm \theta_{0}\right)\right]-\sin ^{2} \nu \eta \sin ^{2}\left[\nu\left(\theta \pm \theta_{0}\right)\right]} d \eta
$$

Using the trigonometric identity $\sin ^{2} x=1-\cos ^{2} x$, the denominator of Eq. (A8) becomes

$$
\begin{aligned}
\text { Denominator }= & (\cosh \nu \eta-1)^{2}+2(\cosh \nu \eta-1)(1 \\
& -\cos \nu \vartheta \cos \nu \pi)+(\cos \nu \pi-\cos \nu \vartheta)^{2},
\end{aligned}
$$

(A9) where

where

$$
R(\eta)=c t=\left[r^{2}+r_{0}^{2}+2 r r_{0} \cos \eta+\left(z-z_{0}\right)^{2}\right]^{1 / 2} .
$$

Substituting Eqs. (A6) and (A7) into Eq. (A5), we obtain

where $\vartheta=\theta \pm \theta_{0}$. By using the relation $S=-\rho / 4 \pi$, we obtain

$$
D_{\eta}=\frac{\cos \nu \pi-\cosh \nu \eta \cos [\nu \vartheta]}{(\cosh \nu \eta-1)^{2}+2(\cosh \nu \eta-1)(1-\cos \nu \vartheta \cos \nu \pi)+(\cos \nu \pi-\cos \nu \vartheta)^{2}} .
$$


A factor of 2 included in Eq. (A10) results from extending the integral from 0 to $-\infty$ by recognizing that the integrant is a even function of $\eta$. Equation (A10), along with Eqs. (A7) and (A11), is exactly the same as Eq. (2a)-(2c).

Abramowitz, M., and Stegun, I. A., eds. (1974). Handbook of Mathematical Functions, With Formulas, Graphs, and Mathematical Tables (Dover, New York).

Biot, M. A., and Tolstoy, I. (1957). "Formulation of wave propagation in infinite media by normal coordinates with an application to diffraction," J. Acoust. Soc. Am. 29, 381-391.

Calamia, P. T. and Svensson, U. P. (2007). "Fast Time-Domain EdgeDiffraction Calculations for Interactive Acoustic Simulations," EURASIP Journal on Advances in Signal Processing, Vol. 2007, pp. 10.

Carslaw, H. S. (1899). "Some multiform solutions of the partial differential equations of physicis and mathematics and their applications," Proc. London Math. Soc. 30, 121-163.

Carslaw, H. S. (1920). "Diffraction of waves by a wedge of any angle," Proc. London Math. Soc. 18, 1291-1306.

Chu, D. (1989). "Impulse response of density contrast wedge using normal coordinates," J. Acoust. Soc. Am. 86, 1883-1896.

Chu, D., and Stanton, T. K. (1998). "Application of pulse compression techniques to broadband acoustic scattering by live individual zooplankton," J. Acoust. Soc. Am. 104, 39-55.

Clay, C. S., Chu, D., and Li, S. (1993). "Specular reflections of transient pressures from finite width plane facets," J. Acoust. Soc. Am. 94, 22792286.

Daneshvar, M. R., and Clay, C. S. (1987). "Imaging of rough surfaces for impulsive and continuously radiating sources," J. Acoust. Soc. Am. 82 , 360-369.

Davis, A. M. J., and Scharstein, R. W. (1997). "The complete extension of the Biot-Tolstoy solution to the density contrast wedge with sample calculations," J. Acoust. Soc. Am. 101, 1821-1835.

Duan, D.-W., Rahmat-Samii, Y., and Mahon, J. P. (1991). "Scattering from a circular disk: A comparative study of PTD and GTD techniques," Proc. IEEE 79, 1472-1480.

Feuillade, C., Chu, D., and Clay, C. S. (2004). "Space-time variations of the acoustic field scattered from a penetrable isovelocity wedge," J. Acoust. Soc. Am. 116, 777-789.

Friedlander, F. G. (1958). Sound Pulses (Cambridge University Press, New York), pp. 108-144.

Gradshteyn, I. S., and Ryzhik, I. M. (1980). Table of Integrals, Series, and Products (Acdemic, Orlando).

Jones, D. S. (1953). "Diffraction by a thick semi-infinite plate," Proc. R. Soc. London 217, 153-175.

Karp, S. N., and Keller, J. B. (1960). "Multiple diffraction by an aperture in a hard screen," Opt. Acta 8, 61-72.

Keiffer, R. S., Novarini, J. C., and Norton, G. V. (1994). "The impulse response of an aperture: Numerical calculations within the framework of the wedge assemblage method," J. Acoust. Soc. Am. 95, 3-12.

Keller, J. B. (1957). "Diffraction by an aperture," J. Appl. Phys. 28, 426444.

Keller, J. B. (1962). "Geometric theory of diffraction," J. Appl. Phys. 52, $116-130$.

Keller, J. B., Lewis, R. M., and Seckler, B. D. (1956). “Asymptotic solution of some diffraction problems," Commun. Pure Appl. Math. 9, 207-265.

Kinney, V. A., Clay, C. S., and Sandness, G. A. (1983). "Scattering from a corrugated surface: Comparison between experiment, Helmholtz-
Kirchhoff theory, and the facet ensemble method," J. Acoust. Soc. Am. 73, 183-194.

Kristensson, G., and Waterman, P. C. (1982). "The T matrix for acoustic and electromagnetic scattering by circular disks," J. Acoust. Soc. Am. 72, $1612-1625$.

Li, L. W., Kooi, P. S., Qiu, Y. L., Yeo, T. S., and Leong, M. S. (1998). "Analysis of electromagnetic scattering of conducting circular disk using a hybrid method," Progress in Electromagnetics Research 20, 101-123. doi: 10.2528/PIER97111200.

Li, S., Chu, D., and Clay, C. S. (1994). "Time domain reflections and diffractions from facet-wedge constructions: Acoustic experiments including double diffractions," J. Acoust. Soc. Am. 96, 3715-2720.

MacDonald, H. M. (1902). Electric Waves (Cambridge University Press, London), pp. 187-198.

MacDonald, H. M. (1915). "A class of diffraction problems," Proc. London Math. Soc. 14, 410-427.

Medwin, H., Childs, E., and Jebsen, G. M. (1982). "Impulse studies of double diffraction: Adiscrete Huygens interpretation," J. Acoust. Soc. Am. 72, 1005-1013.

Medwin, H., and Clay, C. S. (1998). Fundamentals of Acoustic Oceanography (Academic, Boston), Chaps. 11 and 12.

Menounou, P., Busch-Vishniac, I. J., and Blackstock, D. T. (2000). "Directive line source model: A new model for sound diffraction by half planes and wedges," J. Acoust. Soc. Am. 107, 2973-2986.

Morse, P. M., and Ingard, K. U. (1968). Theoretical Acoustics (McGrawHill, New York), pp. 400-466.

Pierce, A. D. (1974). "Diffraction of sound around corners and over wide barriers," J. Acoust. Soc. Am. 55, 941-955.

Pierce, A. D. (1981). Acoustics: An Introduction to its Physical Principles and Applications (McGraw-Hill, New York), pp. 479-501.

Rahmat-Samii, Y. (1988). "Reflector antennas," in Antennas Handbook, edited by Y. T. Lo and S. W. Lee (Rahmat-Samii, a Nostrand-Reinhold Company, New York), Chap. 15.

Sleator, F. B. (1969). The Disc, Ch. 14 in Electromagnetic and Acoustic Scattering by Simple Shapes, edited by J. J. Bowman, T. B. A. Senior, and P. L. E. Uslenghi (Elsevier, New York).

Sommerfeld, A. (1954). Optics: Lectures on Theoretical Physics (Academic, New York), pp. 204-205.

Stanton, T. K., and Chu, D. (2004). "On the acoustic diffraction by the edges of benthic shells," J. Acoust. Soc. Am. 116, 239-244.

Stanton, T. K., Chu, D., and Norton, G. V. (2007). "Acoustic diffraction by deformed edges of finite length: Theory and experiment," J. Acoust. Soc. Am. 122, 3167.

Svensson, U. P., Fred, R. I., and Vanderkooy, J. (1999). "An analytic secondary source model of edge diffraction impulse responses," J. Acoust. Soc. Am. 106, 2331-2344.

Svensson, U.P. and Calamia, P.T. (2006). "Edge-diffraction impulse responses near specular- and shadow-zone boundaries," Acta. Acust. Acust. 92, 501-512.

Tolstoy, I. (1989a). "Diffraction by a hard truncated wedge and a strip," IEEE J. Ocean. Eng. 14, 4-16.

Tolstoy, I. (1989b). "Exact, explicit solutions for diffraction by hard sound barriers and seamounts," J. Acoust. Soc. Am. 85, 661-669.

Tolstoy, I., and Clay, C. S. (1987). Ocean Acoustics: Theory and experiment in underwater sound. (American Institute of Physics, New York), Chap. 3.

Ufimtsev, P. Y. (1962). "Method of edge waves in the physical theory of diffraction," Izd-Vo Sovyetskoye Radio, translated by US Air Force Foreign Tech. Div., 1154 pp. 